\title{
Limit cycles in planar piecewise linear differential systems with nonregular separation line
}

\author{
Pedro Toniol Cardin ${ }^{\mathrm{a}}$, Joan Torregrosa ${ }^{\mathrm{b}, *}$ \\ a Departamento de Matemática, Faculdade de Engenharia de Ilha Solteira, Universidade Estadual Paulista (UNESP), Rua Rio de Janeiro, 266, CEP \\ 15385-000 Ilha Solteira, São Paulo, Brazil \\ ${ }^{\mathrm{b}}$ Departament de Matemàtiques, Universitat Autònoma de Barcelona, 08193 Bellaterra, Barcelona, Spain
}

\section{H I G H L I G H T S}

- The nonregularity in the separation line occurs only in one point.

- The number of periodic orbits is bigger than for the regular case.

- All the periodic orbits have the breaking point in its interior.

- Higher Melnikov theory is used for the described bifurcating phenomena.

- The stabilization phenomena in the number appear increasing the order.

\section{A R T I C L E I N F O}

\section{Article history:}

Received 9 July 2015

Received in revised form

11 July 2016

Accepted 12 July 2016

Available online 19 July 2016

\section{Keywords:}

Non-smooth differential systems in two zones

Limit cycle in Melnikov higher order

perturbation

Nonregular separation line

\begin{abstract}
A B S T R A C T
In this paper we deal with planar piecewise linear differential systems defined in two zones. We consider the case when the two linear zones are angular sectors of angles $\alpha$ and $2 \pi-\alpha$, respectively, for $\alpha \in(0, \pi)$. We study the problem of determining lower bounds for the number of isolated periodic orbits in such systems using Melnikov functions. These limit cycles appear studying higher order piecewise linear perturbations of a linear center. It is proved that the maximum number of limit cycles that can appear up to a sixth order perturbation is five. Moreover, for these values of $\alpha$, we prove the existence of systems with four limit cycles up to fifth order and, for $\alpha=\pi / 2$, we provide an explicit example with five up to sixth order. In general, the nonregular separation line increases the number of periodic orbits in comparison with the case where the two zones are separated by a straight line.
\end{abstract}

(C) 2016 Elsevier B.V. All rights reserved.

\section{Introduction}

Many systems of relevance to applications are modeled using piecewise linear differential systems. The study of such systems goes back to Andronov and coworkers [1] and nowadays still continues receiving attention by many researchers. For more details about piecewise linear (and piecewise smooth in general) differential systems see for instance the books of Filippov [2] and di Bernardo et al. [3] and the references quoted therein.

In the classical theory for smooth systems an important topic is the weak 16 th Hilbert's problem. The question is: Which is the maximum number of isolated periodic orbits, also called limit cycles, that bifurcate perturbing a center? This problem for piecewise differential systems defined in two zones have been studied recently, among other papers, in [4-12]. Usually the separation line between the two zones is a straight line. Here we study the case when the separation line is nonregular. angular regions, i.e. the separation line is formed by two semi-straight lines that coincide at the origin forming an angle $\alpha$, with $\alpha \in(0, \pi)$. In particular we provide lower bounds for the number of limit cycles of the linear center under perturbation, with piecewise linear vector fields, up to order six. After a linear transformation, if it is necessary, it is not restrictive to assume that the center is the classic harmonic oscillator. More precisely, for each

\footnotetext{
* Corresponding author.

E-mail addresses: pedrocardin@gmail.com, pedrocardin@mat.feis.unesp.br (P.T. Cardin), torre@mat.uab.cat (J. Torregrosa).
} 
$N \in \mathbb{N}$, we consider the following piecewise linear perturbation of the linear center

$$
\left\{\begin{array}{l}
\dot{x}=-y+\sum_{i=1}^{N} \varepsilon^{i}\left(a_{0 i}^{ \pm}+a_{1 i}^{ \pm} x+a_{2 i}^{ \pm} y\right), \\
\dot{y}=x+\sum_{i=1}^{N} \varepsilon^{i}\left(b_{0 i}^{ \pm}+b_{1 i}^{ \pm} x+b_{2 i}^{ \pm} y\right),
\end{array}\right.
$$

defined in the angular regions separated by the line $\Sigma_{\alpha}$. In fact the separation line $\Sigma_{\alpha}$ is defined as follows. For $\alpha \in(0, \pi)$ and $\alpha \neq \pi / 2$, then $\Sigma_{\alpha}=\{(x, y): x \geq 0, y=0\} \cup\left\{(x, y): x=(\tan \alpha)^{-1} y, y \geq 0\right\}$. For $\alpha=\pi / 2$, we have $\Sigma_{\frac{\pi}{2}}=\{(x, y): x \geq 0, y=0\} \cup\{(x, y):$ $x=0, y \geq 0\}$. Finally, $\Sigma_{\pi}$ denotes the straight line $\{(x, y): y=0\}$. The notations $\Sigma_{\alpha}^{ \pm}$indicate the angular sectors of angles $\alpha$ and $2 \pi-\alpha$ separated by $\Sigma_{\alpha}$, respectively. We denote the vector fields associated to system (1), defined in $\Sigma_{\alpha}^{ \pm}$, by $X^{ \pm}$, respectively. The point (0, 0 ) where the separation line $\Sigma_{\alpha}$ loses its regularity will be referred to as the breaking point.

It is worth to emphasize that, with the perturbations that we have considered, the perturbed systems do not escape from the class of piecewise linear system, but we consider the period annulus of the center instead of a neighborhood of the origin. This is the aim of the higher order Poincaré-Pontryagin-Melnikov theory instead of degenerated Hopf bifurcation. This theory provides the same results, in the plane, than the averaging one. In this paper, $N$ denotes the degree in the perturbation parameter $\varepsilon$, or the order of perturbation in $\varepsilon$.

The number of limit cycles close to the origin for piecewise families, using Lyapunov constants, is studied in [13,12]. All the families introduced in both works have the origin as a critical point for the systems defined in $\Sigma_{\pi}^{ \pm}$, respectively. In fact, the perturbations are of higher order in the variables. In our case the perturbations are linear in the variables but nonlinear in the parameter $\varepsilon$. Moreover, we do not preserve the origin as a critical point in $\Sigma_{\alpha}^{ \pm}$, then the technique used in those papers, based on a change to polar coordinates, is more difficult to apply. Consequently these two problems are not equivalent.

In the case when the separation line is a straight line, Han and Zhang in [7] conjectured that the maximum number of limit cycles for planar discontinuous piecewise linear systems should be at most two. However, Huan and Yang in [8] provided strong numerical evidence that three limit cycles should exist. A computer-assisted proof of the existence of such limit cycles was given in [10]. The existence of other examples with three limit cycles, via bifurcation techniques, can be found in [4,14]. The example given in [4] uses a piecewise linear perturbation of a linear center and it is proved that three is the maximum number of limit cycles that can appear up to a seventh order perturbation. Moreover, as was observed in [4], when the order of the perturbation increases, the number of limit cycles seems to stabilize in three. However, it is still an open question to determine whether three is the maximum number of limit cycles for planar discontinuous piecewise linear differential systems when the separation line is a straight line. In this case, Euzébio and Llibre in [15] proved that if one of the linear differential systems has its equilibrium point on the straight line of separation, then the maximum number is less than or equal to four. This upper bound is decreased by two in the same cases in [16,17]. For this special class, the complete study is done in [18], where it is shown that the maximum number of limit cycles is two. Moreover, this upper bound is reached.

When the separation line is no longer a straight line, it is possible to obtain more than three limit cycles. Braga and Mello in [19] showed the importance of the separation boundary in the number of bifurcated limit cycles. They proved the existence of piecewise linear differential systems with two zones in the plane with four, five, six and seven limit cycles, and conjectured that, given $n \in \mathbb{N}$, there is a piecewise linear system with two zones in the plane with exactly $n$ limit cycles. Promptly, Novaes and Ponce in [20] gave a positive answer to this conjecture. Braga and Mello in [21] also showed the existence of a class of discontinuous piecewise linear differential systems with two zones in the plane having exactly $n$ hyperbolic limit cycles. As it was pointed out in [21], in the obtained examples in [20], the limit cycles can be nonhyperbolic.

In this article, we highlight once again the importance of the separation line and the number of breaking points in the number of limit cycles that can appear by perturbation in piecewise linear vector fields. We study the bifurcation of limit cycles by studying higher order piecewise linear perturbations of a linear center. We follow the procedure described in [4] to study the $\Sigma_{\alpha}$-piecewise linear vector field and we get four limit cycles for every $\alpha \in(0, \pi)$ and five for $\alpha=\pi / 2$. This shows that, in general, one can obtain more limit cycles in comparison with the case of $\Sigma_{\pi}$-piecewise linear vector fields. Clearly, the functions to be studied, which ensure the existence of all these limit cycles, cannot be well defined when $\alpha$ goes to $\pi$ or to 0 . We will come back to this question later.

In the works $[19,20]$ as well as in the present paper, all the considered limit cycles are nested and they intersect $\Sigma_{\alpha}$ only at crossing points. That is, the limit cycles intersecting the sliding region are not considered. Below we give the precise definitions. Besides the method used, the main qualitative difference with [19] is that we have only one breaking point which defines the nonregular set in $\Sigma_{\alpha}$, and not one between two consecutive limit cycles. Moreover all our limit cycles have this breaking point in its interior. With respect to [20] we observe that the separation line is analytic.

For analytic vector fields the number of limit cycles usually increases when higher order perturbations are considered. It is well known that, up to a first order analysis in $\varepsilon$, perturbing the linear center with arbitrary polynomials of degree $n$, we can only obtain [ $n-1) / 2$ ] limit cycles for the perturbed system, where [·] denotes the integer part function, see [22]. On the other hand but in the same class of systems, in [23] it is proved that the maximum number of limit cycles is lower than or equal to [N( $n-1) / 2]$. This upper bound, in general, is reached when $n$ is large enough and $N=2$. In many classes of polynomial systems, when $N$ increases, the number of limit cycles usually stabilizes. The stabilization process depends on the considered family. In [24] this phenomenon is studied for some families. For example, a concrete class is presented such that the maximum number of limit cycles is $0,0,1,1,1,2,2,2,2,2$ when $N=1, \ldots, 10$. In [23], considering perturbations of a linear center by quadratic polynomials, it is shown that when $N=1, \ldots, 6$, the maximum number of limit cycles is $0,1,1,2,2,3$, respectively. A higher order study is not necessary because Bautin in [25], for quadratic systems, proves that at most three limit cycles can appear near a focus or a center. This stabilization phenomenon also appears in piecewise linear systems. In [4] it is proved that for system (1), with separation line $\Sigma_{\pi}$, the maximum number of limit cycles is $1,1,2,3,3,3,3$ when $N=1,2,3,4,5,6,7$, respectively. In this paper we have not shown if the stabilization procedure also appears in general piecewise linear systems with nonregular separation line because of the computations. But we think that this phenomenon will appear for every family of systems, as we show in Section 5 for some classes of $\Sigma_{\alpha}$-piecewise linear Liénard systems. 
a

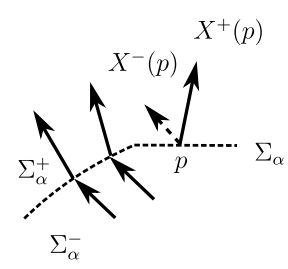

b

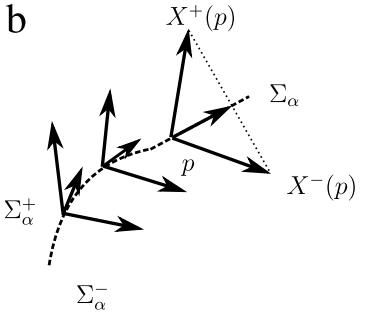

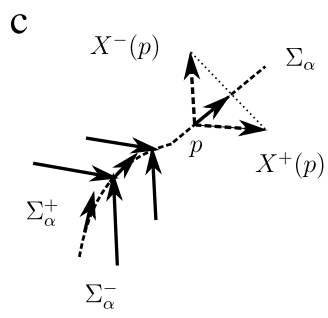

Fig. 1. Filippov convention for (a) crossing, (b) escaping and (c) sliding regions
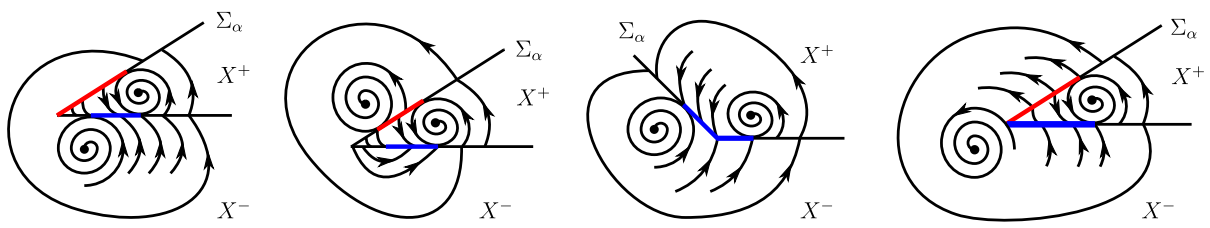

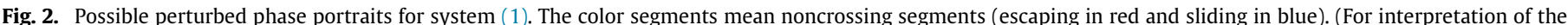
references to color in this figure legend, the reader is referred to the web version of this article.)

Before the presentation of our main results we introduce some definitions and notation. In our approach, Filippov convention [2] is considered. Let $X=\left(X^{+}, X^{-}\right)$be a general piecewise vector field with nonregular boundary $\Sigma_{\alpha}$. We assume that $(0,0)$ is the only breaking point. The points on $\Sigma_{\alpha}^{*}=\Sigma_{\alpha} \backslash\{(0,0)\}$ where both vector fields $X^{+}$and $X^{-}$simultaneously point outward or inward for $\Sigma_{\alpha}^{*}$ define the escaping and sliding regions. The complement of these regions in $\Sigma_{\alpha}^{*}$, excluding the tangency points of $X^{ \pm}$with $\Sigma_{\alpha}$, defines the crossing (or sewing) region. See these situations in Fig. 1. If the boundary between the two zones is regular (i.e. $\Sigma_{\alpha}$ is a smooth curve), the definitions of crossing, escaping and sliding regions make sense for any $p \in \Sigma_{\alpha}$.

Any segment $S$ contained in an escaping or a sliding region is called usually a sliding segment. A periodic orbit that intersects $\Sigma_{\alpha}$ but not the escaping or sliding region is called $\Sigma_{\alpha}$-crossing periodic orbit. When this periodic orbit is isolated, we call it $\Sigma_{\alpha}$-crossing limit cycle, or simply crossing limit cycle. In this paper we only study this type of periodic orbits bifurcating from the linear center. In the case when the separation line is the straight line $\Sigma_{\pi}$, for $\varepsilon$ small enough, the sliding segment of system (1) is an open interval that contains the origin. However, if the separation line is no longer a straight line, system (1) can have more than one sliding segment separated by the breaking point. Examples of this situation can be seen in Fig. 2. Additionally, for $\varepsilon$ small enough in (1), the critical point of $X^{+}$and $X^{-}$is a focus or a center.

This work is an application of a generalization of Françoise's method for smooth systems, see [26]. More specifically, the method used in this work, see [27], is a generalization to piecewise linear systems of the extension to higher order perturbations, see [28], of the method of Françoise. The main application in [27] is the computation of the Lyapunov constants for piecewise systems and their use in the centerfocus problem. Other applications of this method can be found in $[29,30]$. This procedure is useful not only to discuss the weak 16 th Hilbert's problem, but also to study related problems such that the persistence of centers under small perturbations, and the study of the period function for centers, see [4].

The method described in [27] is based on a decomposition of certain one-forms associated to the expression of the vector field in polar coordinates. The decomposition, see Section 2, is done in such a way that it simplifies the computations of the first nonzero term, $M_{N}(\rho)$, of the expansion in $\varepsilon$ of the return map associated to the vector field defined on the positive $x$-axis, so that

$$
M(\rho, \varepsilon)=\rho+\varepsilon^{N} M_{N}(\rho)+O\left(\varepsilon^{N+1}\right) .
$$

In this case the function $M_{N}(\rho)$ is called the first nonvanishing Poincaré-Pontryagin-Melnikov function. As in smooth systems, for each simple zero $\rho_{0}$ of $M_{N}(\rho)$, there exists a hyperbolic limit cycle $\gamma_{\varepsilon}$ of the perturbed system (1), such that $\gamma_{\varepsilon}$ goes to $\gamma_{0}$ when $\varepsilon$ goes to 0 , where $\gamma_{0}$ is the level curve $\left\{x^{2}+y^{2}=\rho_{0}^{2}\right\}$ of the unperturbed system. When $M_{i}=0$ for $i=1, \ldots, N-1$, then the number of zeros of $M_{N}(\rho)$ determines the upper bound of the number of limit cycles bifurcating from the center of the unperturbed system up to order $N$. Our main result is the following.

Theorem 1.1. For system (1) and $\alpha \in(0, \pi)$, the maximum number, $Z_{N}$, of zeros of the corresponding function $M_{N}(\rho)$ is $1,2,2,3,4,5$ when $N=1, \ldots, 6$. Moreover, for each $N \leq 5$, there exist perturbation parameters such that system (1), for $\varepsilon$ small enough, exhibits $Z_{N}$ hyperbolic limit cycles for every $\alpha \in(0, \pi)$. In addition, when $\alpha=\pi / 2$, for some concrete perturbation parameters and for $\varepsilon$ small enough, system ( 1$)$ has five limit cycles.

We recall that, in [4], the authors proved that system (1), when the separation line is $\Sigma_{\pi}$, has three limit cycles. In order to compare with this regular case, a natural question appears: What happens with the extra limit cycles, when they exist, that appear in Theorem 1.1 when $\alpha$ goes to $\pi$ ? Analyzing the functions $M_{N}(\rho)$ in Section 3, it is possible to show that the radius of at least one of the limit cycles goes to infinity. Consequently at least one limit cycle goes to infinity when $\alpha$ goes to $\pi$. Moreover, if $\alpha$ goes to 0 the system becomes linear so there are no limit cycles. In this case all the limit cycles go to origin. See Remark 3.3.

This paper is organized as follows. In Section 2 we provide the main tools to prove the results of this work. In Section 3 we study the maximum number of limit cycles that appears from $\Sigma_{\alpha}$-piecewise linear perturbations of a linear center up to a sixth order perturbation. In addition, we prove the existence of a piecewise linear system with four $\Sigma_{\alpha}$-crossing limit cycles. For $\alpha=\pi / 2$, see Section 4 , we give an explicit example with five limit cycles, together with the numerical simulation for small values of $\varepsilon$. Moreover we also get the values of $\varepsilon$ where some of these limit cycles disappear in a semistable limit cycle bifurcation. In Section 5 we show some piecewise linear Liénard systems where the stabilization process, commented previously, appears. We finish with a concluding section. Although the computations 


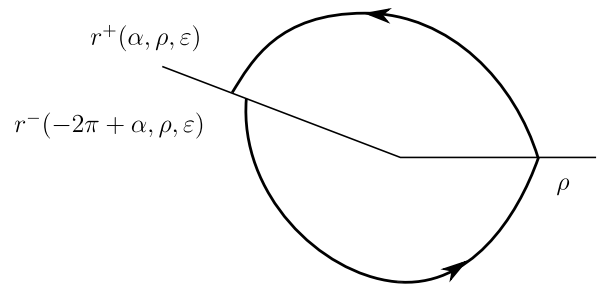

Fig. 3. Difference of the half-return maps of system (1).

of this work can be done analytically, due to the size of the expressions that appear in some of the proofs, almost all have been done with a Computer Algebra System. ${ }^{1}$

\section{Difference map for $\Sigma_{\alpha}$-piecewise linear systems}

This section is devoted to present the main tools that we need to state and prove the results of this paper. We extend to $\Sigma_{\alpha}$-piecewise linear vector fields the presentation given in [4] for the $\Sigma_{\pi}$ case. The method described uses the decomposition of a one-form given in [26,32] but for higher order perturbations, in polar coordinates, as was introduced in [27].

Consider a system

$$
X^{ \pm}:(\dot{x}, \dot{y})=\left(-y+\sum_{i=1}^{N} \varepsilon^{i} P_{i}^{ \pm}(x, y), x+\sum_{i=1}^{N} \varepsilon^{i} Q_{i}^{ \pm}(x, y)\right)
$$

where $P_{i}^{ \pm}(x, y)$ and $Q_{i}^{ \pm}(x, y)$ are analytic functions, defined in the regions $\Sigma_{\alpha}^{ \pm}$, see (1). The above vector field $X^{ \pm}$, in polar coordinates $(x, y)=(r \cos \theta, r \sin \theta)$, writes as

$$
(\dot{r}, \dot{\theta})= \begin{cases}\left(\sum_{i=1}^{N} \varepsilon^{i} R_{i}^{+}(r, \theta), 1+\sum_{i=1}^{N} \varepsilon^{i} \Theta_{i}^{+}(r, \theta)\right) & \text { if } \theta \in[0, \alpha), \\ \left(\sum_{i=1}^{N} \varepsilon^{i} R_{i}^{-}(r, \theta), 1+\sum_{i=1}^{N} \varepsilon^{i} \Theta_{i}^{-}(r, \theta)\right) & \text { if } \theta \in[\alpha, 2 \pi),\end{cases}
$$

where $R_{i}^{ \pm}, \Theta_{i}^{ \pm}$are analytic functions in $r, \sin \theta$, and $\cos \theta$. Then it can also be expressed as

$$
\begin{cases}d H+\sum_{i=1}^{N} \varepsilon^{i} \omega_{i}^{+}=0 & \text { if } \theta \in[0, \alpha), \\ d H+\sum_{i=1}^{N} \varepsilon^{i} \omega_{i}^{-}=0 & \text { if } \theta \in[\alpha, 2 \pi),\end{cases}
$$

where $H(r)=\left(x^{2}+y^{2}\right) / 2=r^{2} / 2$, and $\omega_{i}^{ \pm}=\omega_{i}^{ \pm}(r, \theta)$ are analytic one-forms, $2 \pi$-periodic in $\theta$ and polynomial in $r$.

Let $r^{+}(\theta, \rho, \varepsilon)$ (resp. $\left.r^{-}(\theta, \rho, \varepsilon)\right)$ be the solution of $X^{ \pm}$such that $r^{+}(0, \rho, \varepsilon)=\rho$ (resp. $r^{-}(0, \rho, \varepsilon)=\rho$ and reversing the angle $\theta$ ). Observe that, for $\varepsilon$ small enough, it is well defined in an annular region that does not contain the origin. The periodic orbits are the zeros of the difference map $\Delta_{\alpha}(\rho, \varepsilon)=r^{+}(\alpha, \rho, \varepsilon)-r^{-}(-2 \pi+\alpha, \rho, \varepsilon)$, see Fig. 3 .

We write the solution $r^{ \pm}(\theta, \rho, \varepsilon)$ in power series of $\varepsilon$ as $r^{ \pm}(\theta, \rho, \varepsilon)=\sum_{i=0}^{N} r_{i}^{ \pm}(\theta, \rho) \varepsilon^{i}$. It can be checked easily that $r^{ \pm}(\theta, \rho, 0)=$ $r_{0}^{ \pm}(\theta, \rho) \equiv \rho$ and $r_{i}^{ \pm}(0, \rho) \equiv 0$. Next proposition provides the first nonvanishing term of the difference map $\Delta_{\alpha}(\rho, \varepsilon)$ in series of $\varepsilon$ and the relation between its simple zeros with the limit cycles of $(2)$.

Proposition 2.1. Denoting by $\widehat{M}_{N}(\rho)=r_{N}^{+}(\alpha, \rho)-r_{N}^{-}(-2 \pi+\alpha, \rho)$, the Poincaré-Pontryagin-Melnikov functions of order $N$ for system (2) are given by

$$
M_{1}(\rho)=\widehat{M}_{1}(\rho) \quad \text { and } \quad M_{N}(\rho)=\left.\widehat{M}_{N}(\rho)\right|_{\left\{M_{k}(\rho) \equiv 0, k=1, \ldots, N-1\right\}}, \quad \text { for } N \geq 2 .
$$

Moreover, if $\rho$ is a simple zero of $M_{N}$, then a limit cycle of system (2) exists and it converges to the level curve $x^{2}+y^{2}=\rho^{2}$ when $\varepsilon$ goes to zero.

The functions $M_{N}(\rho)$ depend on the coefficients of the perturbations. But they only coincides with the Poincaré-Pontryagin-Melnikov functions when the previous ones vanish identically. In the sequel we describe how these functions can be computed. The explicit expressions of $r_{i}(\theta, \rho)$ are given recursively in Theorem 2.3 and explicitly in Corollary 2.4. The functions $F, S$, and $h$ that appear in those statements are obtained from the decomposition defined in Lemma 2.2. In particular, Propositions 2.5 and 2.6 and Corollary 2.7 give how these functions can be written for piecewise linear systems. Finally, a concrete example, for which we show the procedure to obtain the first nonvanishing terms of its return map, is given in Proposition 2.8.

\footnotetext{
1 The computations are done with MAPLE ${ }^{\mathrm{TM}}$ in a Xeon computer (CPU E5-450, 3.0 GHz, RAM 32 Gb) with GNU Linux. See [31].
} 
Consider now initial the value problem

$$
\left\{\begin{array}{l}
d H+\sum_{i=1}^{N} \varepsilon^{i} \omega_{i}=0 \\
r(0, \rho, \varepsilon)=\rho
\end{array}\right.
$$

where $H(r)=r^{2} / 2$ and $\omega_{i}=\omega_{i}(r, \theta)$ are smooth one-forms $2 \pi$-periodic in $\theta$. The solution $r(\theta, \rho, \varepsilon)$ of $(3)$ writes as $r(\theta, \rho, \varepsilon)=$ $\sum_{i=0}^{N} r_{i}(\theta, \rho) \varepsilon^{i}$ and it can be checked easily that $r(\theta, \rho, 0)=r_{0}(\theta, \rho) \equiv \rho$.

Lemma $2.2([27])$. Let $\Omega=\alpha(r, \theta) d r+\beta(r, \theta) d \theta$ be an arbitrary analytic one-form, $2 \pi$-periodic in $\theta$, and $H(r)=r^{2} / 2$. Then there exist functions $h(r, \theta), S(r, \theta)$ and $F(r)$ also $2 \pi$-periodic in $\theta$ and defined by $F(r)=\frac{1}{2 \pi} \int_{0}^{2 \pi} \beta(r, \psi) d \psi, S(r, \theta)=\int_{0}^{\theta} \beta(r, \psi) d \psi-F(r) \theta$ and $h(r, \theta)=\left(\alpha(r, \theta)-\frac{\partial S(r, \theta)}{\partial r}\right) / H^{\prime}(r)$, such that

$$
\Omega=\Omega^{0}+\Omega^{1} \text { where } \Omega^{0}=h d H+d S, \Omega^{1}=F(r) d \theta,
$$

and

$$
\int_{H=\rho^{2} / 2} \Omega^{0}=0, \quad \int_{H=\rho^{2} / 2} \Omega^{1}=\int_{H=\rho^{2} / 2} \Omega .
$$

Theorem 2.3 ([27]). Let $r(\theta, \rho, \varepsilon)$ be the solution of (3). Then for any $n \in \mathbb{N}, r(\theta, \rho, \varepsilon)$ satisfies the following implicit equation

$$
\frac{r^{2}(\theta, \rho, \varepsilon)-\rho^{2}}{2}+O\left(\varepsilon^{n+1}\right)=\sum_{i=1}^{n} \varepsilon^{i}\left[\int_{0}^{\theta} F_{i}(r(\psi, \rho, \varepsilon)) d \psi+\left.S_{i}(r(\psi, \rho, \varepsilon), \psi)\right|_{\psi=0} ^{\psi=\theta}\right],
$$

where the one-forms $\Omega_{i}$ and the functions $F_{i}(r), h_{i}(r, \theta)$ and $S_{i}(r, \theta)$ are defined inductively as follows: $h_{0}=1$,

$$
-\Omega_{1}:=-\omega_{1} h_{0}=h_{1} d H+d S_{1}+F_{1} d \theta,
$$

and

$$
-\Omega_{i}:=-\sum_{j=1}^{i} \omega_{j} h_{i-j}=h_{i} d H+d S_{i}+F_{i} d \theta
$$

for $i=2, \ldots, n$ using the decomposition given in Lemma 2.2 for the one-forms $-\Omega_{i}$.

From the above theorem we can obtain recursively the expressions of $r_{n}(\theta, \rho)$. Next result provides their explicit expressions up to sixth order.

Corollary 2.4. Let $r(\theta, \rho, \varepsilon)=\sum_{i=0}^{N} r_{i}(\theta, \rho) \varepsilon^{i}$ be the solution of (3). Assume that the functions $r_{0}(\theta, \rho)=\rho, r_{1}(\theta, \rho), r_{2}(\theta, \rho)$, . , $r_{n-1}(\theta, \rho)$ are known. Then $r_{n}(\theta, \rho)$ can be obtained equating the terms of order $\varepsilon^{n}$ in both sides of Eq. (4). In fact, this equality writes

$$
\rho r_{n}(\theta, \rho)=\mathcal{F}_{n}\left(\theta, \rho, r_{1}, \ldots, r_{n-1}\right),
$$

where $\mathcal{F}_{n}$ depends on the one-forms $\omega_{1}, \omega_{2}, \ldots, \omega_{n}$, through the corresponding $F_{i}, S_{i}$ and $r_{i}=r_{i}(\theta, \rho)$ for $i=1,2, \ldots, n$. In particular,

$$
\begin{aligned}
& \mathcal{F}_{1}=S_{1}+F_{1} \theta \text {, } \\
& \mathcal{F}_{2}=-\frac{1}{2} r_{1}^{2}+S_{2}+D_{1}\left(S_{1}\right) r_{1}+F_{2} \theta+F_{1}^{\prime} I\left(r_{1}\right) \text {, } \\
& \mathcal{F}_{3}=-r_{1} r_{2}+S_{3}+r_{1} D_{1}\left(S_{2}\right)+\frac{1}{2} r_{1}^{2} D_{1}^{2}\left(S_{1}\right)+r_{2} D_{1}\left(S_{1}\right)+F_{3} \theta+\frac{1}{2} F_{1}^{\prime \prime} I\left(r_{1}^{2}\right)+F_{1}^{\prime} I\left(r_{2}\right)+F_{2}^{\prime} I\left(r_{1}\right), \\
& \mathcal{F}_{4}=-r_{1} r_{3}-\frac{1}{2} r_{2}^{2}+S_{4}+r_{1} D_{1}\left(S_{3}\right)+\frac{1}{2} r_{1}^{2} D_{1}^{2}\left(S_{2}\right)+r_{2} D_{1}\left(S_{2}\right)+\frac{1}{6} r_{1}^{3} D_{1}^{3}\left(S_{1}\right) \\
& +r_{1} r_{2} D_{1}^{2}\left(S_{1}\right)+r_{3} D_{1}\left(S_{1}\right)+F_{4} \theta+F_{3}^{\prime} I\left(r_{1}\right)+\frac{1}{2} F_{2}^{\prime \prime} I\left(r_{1}^{2}\right)+F_{2}^{\prime \prime} I\left(r_{2}\right)+\frac{1}{6} F_{1}^{(3)} I\left(r_{1}^{3}\right)+F_{1}^{\prime \prime} I\left(r_{1} r_{2}\right)+F_{1}^{\prime} I\left(r_{3}\right), \\
& \mathcal{F}_{5}=r_{1} r_{4}-r_{2} r_{3}+S_{5}+r_{1} D_{1}\left(S_{4}\right)+\frac{1}{2} r_{1}^{2} D_{1}^{2}\left(S_{3}\right)+r_{2} D_{1}\left(S_{3}\right)+\frac{1}{6} r_{1}^{3} D_{1}^{3}\left(S_{2}\right)+r_{1} r_{2} D_{1}^{2}\left(S_{2}\right) \\
& +r_{3} D_{1}\left(S_{2}\right)+\frac{1}{24} r_{1}^{4} D_{1}^{4}\left(S_{1}\right)+\frac{1}{2} r_{1}^{2} r_{2} D_{1}^{3}\left(S_{1}\right)+r_{1} r_{3} D_{1}^{2}\left(S_{1}\right)+\frac{1}{2} r_{2}^{2} D_{1}^{2}\left(S_{1}\right)+r_{4} D_{1}\left(S_{1}\right) \\
& +F_{5} \theta+F_{4}^{\prime} I\left(r_{1}\right)+\frac{1}{2} F_{3}^{\prime \prime} I\left(r_{1}^{2}\right)+F_{3}^{\prime} I\left(r_{2}\right)+\frac{1}{6} F_{2}^{(3)} I\left(r_{1}^{3}\right)+F_{2}^{\prime \prime} I\left(r_{1} r_{2}\right)+F_{2}^{\prime} I\left(r_{3}\right) \\
& +\frac{1}{24} F_{1}^{(4)} I\left(r_{1}^{4}\right)+\frac{1}{2} F_{1}^{(3)} I\left(r_{1}^{2} r_{2}\right)+F_{1}^{\prime \prime} I\left(r_{1} r_{3}\right)+\frac{1}{2} F_{1}^{\prime \prime} I\left(r_{2}^{2}\right)+F_{1}^{\prime} I\left(r_{4}\right), \\
& \mathcal{F}_{6}=-r_{1} r_{5}-r_{2} r_{4}-\frac{1}{2} r_{3}^{2}+S_{6}+r_{1} D_{1}\left(S_{5}\right)+\frac{1}{2} r_{1}^{2} D_{1}^{2}\left(S_{4}\right)+r_{2} D_{1}\left(S_{4}\right)+\frac{1}{6} r_{1}^{3} D_{1}^{3}\left(S_{3}\right) \\
& +\frac{1}{2} r_{2}^{2} D_{1}^{2}\left(S_{2}\right)+r_{1} r_{2} D_{1}^{2}\left(S_{3}\right)+r_{3} D_{1}\left(S_{3}\right)+\frac{1}{24} r_{1}^{4} D_{1}^{4}\left(S_{2}\right)+\frac{1}{2} r_{1}^{2} r_{2} D_{1}^{3}\left(S_{2}\right) \\
& +r_{1} r_{3} D_{1}^{2}\left(S_{2}\right)+r_{4} D_{1}\left(S_{2}\right)+\frac{1}{120} r_{1}^{5} D_{1}^{5}\left(S_{1}\right)+\frac{1}{6} r_{1}^{3} r_{2} D_{1}^{4}\left(S_{1}\right)+\frac{1}{2} r_{1}^{2} r_{3} D_{1}^{3}\left(S_{1}\right) \\
& +\frac{1}{2} r_{1} r_{2}^{2} D_{1}^{3}\left(S_{1}\right)+r_{1} r_{4} D_{1}^{2}\left(S_{1}\right)+r_{2} r_{3} D_{1}^{2}\left(S_{1}\right)+r_{5} D_{1}\left(S_{1}\right)+F_{6} \theta+F_{5}^{\prime} I\left(r_{1}\right)
\end{aligned}
$$




$$
\begin{aligned}
& +\frac{1}{2} F_{4}^{\prime \prime} I\left(r_{1}^{2}\right)+F_{4}^{\prime} I\left(r_{2}\right)+\frac{1}{6} F_{3}^{(3)} I\left(r_{1}^{3}\right)+F_{3}^{\prime \prime} I\left(r_{1} r_{2}\right)+F_{3}^{\prime} I\left(r_{3}\right)+\frac{1}{24} F_{2}^{(4)} I\left(r_{1}^{4}\right) \\
& +\frac{1}{2} F_{2}^{(3)} I\left(r_{1}^{2} r_{2}\right)+F_{2}^{\prime \prime} I\left(r_{1} r_{3}\right)+\frac{1}{2} F_{2}^{\prime \prime} I\left(r_{2}^{2}\right)+F_{2}^{\prime} I\left(r_{4}\right)+\frac{1}{120} F_{1}^{(5)} I\left(r_{1}^{5}\right)+\frac{1}{6} F_{1}^{(4)} I\left(r_{1}^{3} r_{2}\right) \\
& +\frac{1}{2} F_{1}^{(3)} I\left(r_{1}^{2} r_{3}\right)+\frac{1}{2} F_{1}^{(3)} I\left(r_{1} r_{2}^{2}\right)+F_{1}^{\prime \prime} I\left(r_{1} r_{4}\right)+F_{1}^{\prime \prime} I\left(r_{2} r_{3}\right)+F_{1}^{\prime} I\left(r_{5}\right) .
\end{aligned}
$$

Here $f^{(i)}=\left.\frac{d^{i} f(r)}{d r^{i}}\right|_{r=\rho}, D_{1}^{(i)}(f)=\left.\frac{\partial^{i} f(r, \theta)}{\partial r^{i}}\right|_{r=\rho}$, and $I(f)=\int_{0}^{\theta} f(\psi, \rho) d \psi$.

We remark that in general, from (5), we cannot extend the return map to the origin. More precisely the functions $\widehat{M}_{i}(\rho)$, in Proposition 2.1, can have a singularity at the origin. This fact is shown in the example described in Proposition 2.8. Next two propositions, proved in [4], provide the expressions for $F_{i}, h_{i}$, and $S_{i}$ for piecewise linear systems.

Proposition 2.5. Consider the system

$$
(\dot{x}, \dot{y})=\left(-y+\sum_{i=1}^{N} \varepsilon^{i}\left(a_{0 i}+a_{1 i} x+a_{2 i} y\right), x+\sum_{i=1}^{N} \varepsilon^{i}\left(b_{0 i}+b_{1 i} x+b_{2 i} y\right)\right) .
$$

For any $n \in \mathbb{N}$, the functions $F_{i}(r), S_{i}(r, \theta)$ and $h_{i}(r, \theta)$, for $i=1, \ldots, n$, provided by Theorem 2.3, can be rewritten in the following form: $F_{i}(r)=f_{i} r^{2}, S_{i}(r, \theta)=p_{1, i}(\theta) r+p_{2, i}(\theta) r^{2}$ and $h_{i}(r, \theta)=g_{i}$, for $i=1, \ldots, n$, with $f_{i}$ and $g_{i}$ real numbers and $p_{k, i}$ homogeneous trigonometric polynomials of degree $k$.

Proposition 2.6. For any $n \in \mathbb{N}$, given the functions $F_{i}(r)=f_{i} r^{2}, S_{i}(r, \theta)=p_{1, i}(\theta) r+p_{2, i}(\theta) r^{2}$ and $h_{i}(r, \theta)=g_{i}$, for $i=1, \ldots, n$, obtained in Proposition 2.5 and the functions $r_{i}(\theta, \rho)$, for $i=1, \ldots, n-1$, we have

$$
\begin{aligned}
r_{n}(\theta, \rho)= & \rho\left[f_{n} \theta+p_{2, n}(\theta)-p_{2, n}(0)\right]+\left[p_{1, n}(\theta)-p_{1, n}(0)+2 \sum_{j=1}^{n-1}\left(p_{2, n-j}(\theta) r_{j}(\theta, \rho)+f_{n-j} \int_{0}^{\theta} r_{j}(\psi, \rho) d \psi\right)\right] \\
& +\frac{1}{\rho} \sum_{j=1}^{n-1}\left[-\frac{1}{2} r_{j}(\theta, \rho) r_{n-j}(\theta, \rho)+p_{1, n-j}(\theta) r_{j}(\theta, \rho)\right. \\
& \left.+\sum_{k=1}^{j-1}\left(p_{2, n-j}(\theta) r_{k}(\theta, \rho) r_{j-k}(\theta, \rho)+f_{n-j} \int_{0}^{\theta} r_{k}(\psi, \rho) r_{j-k}(\psi, \rho) d \psi\right)\right] .
\end{aligned}
$$

We can use Propositions 2.5 and 2.6 to obtain, recursively, the explicit expressions for $r_{i}(\alpha, \rho)$ and $r_{i}(-2 \pi+\alpha, \rho)$. Due to the size of them, next corollary provides only the first two.

Corollary 2.7. Let $r(\theta, \rho, \varepsilon)=\sum_{i=1}^{N} r_{i}(\theta, \rho) \varepsilon^{i}$ be the solution of system (6), written in polar coordinates. Then,

$$
\begin{aligned}
r_{1}(\alpha, \rho)= & b_{01}(1-\cos \alpha)+a_{01} \sin \alpha+\left[\left(a_{11}+b_{21}\right) \alpha+\left(a_{21}+b_{11}\right) \sin ^{2} \alpha+\left(a_{11}-b_{21}\right) \cos \alpha \sin \alpha\right] \rho / 2, \\
r_{1}(-2 \pi+\alpha, \rho)= & r_{1}(\alpha, \rho)-\pi\left(a_{11}+b_{21}\right) \rho \\
r_{2}(\alpha, \rho)= & \left.\frac{1}{2}\left(a_{01}^{2}-b_{01}^{2}\right) \sin ^{2} \alpha-a_{01} b_{01} \sin \alpha \cos \alpha\right] \rho^{-1}+\left[b_{02}+a_{01}\left(a_{11}+b_{21}\right)\right. \\
& -b_{01} b_{11}+\frac{1}{2} b_{01}\left(a_{11}+b_{21}\right) \alpha+\left(a_{02}+a_{01} a_{21}-b_{01}\left(a_{11}+b_{21}\right)\right) \sin \alpha \\
& +\left(-b_{02}-a_{01}\left(a_{11}+b_{21}\right)+b_{01} b_{11}\right) \cos \alpha+\frac{1}{2} b_{01}\left(a_{11}-b_{21}\right) \sin \alpha \cos \alpha \\
& +\frac{1}{2} b_{01}\left(a_{21}+b_{11}\right) \sin ^{2} \alpha+\left(a_{01}\left(a_{11}-b_{21}\right)-b_{01}\left(a_{21}+b_{11}\right)\right) \sin ^{2} \alpha \cos \alpha \\
& \left.-\left(a_{01}\left(a_{21}+b_{11}\right)+b_{01}\left(a_{11}-b_{21}\right)\right) \sin \alpha \cos ^{2} \alpha\right]+\left[\frac { 1 } { 8 } \left(4 a_{22}+4 b_{12}\right.\right. \\
& \left.+a_{21} b_{11}-b_{11}^{2}+3 a_{21}^{2}+2 a_{11}^{2}-2 b_{21}^{2}\right)+\frac{1}{8}\left(a_{11}+b_{21}\right)^{2} \alpha^{2}+\frac{1}{2}\left(a_{12}+b_{22}\right. \\
& \left.+a_{21}\left(a_{11}+b_{21}\right)\right) \alpha-\frac{1}{4}\left(a_{11}+b_{21}\right)\left(a_{21}+b_{11}\right) \alpha \cos ^{2} \alpha+\left(\frac{1}{4}\left(a_{11}^{2}-b_{21}^{2}\right) \alpha\right. \\
& \left.+\frac{1}{2}\left(a_{11} a_{21}-b_{11} b_{21}-2 a_{21} b_{21}+a_{12}-b_{22}\right)\right) \sin \alpha \cos _{\alpha}+\frac{1}{8}\left(a_{11}^{2}-4 b_{12}\right. \\
& \left.-2 b_{11}^{2}-6 a_{21}^{2}+5 b_{21}^{2}-4 a_{22}-8 a_{21} b_{11}-6 a_{11} b_{21}\right) \cos ^{2} \alpha+\frac{3}{4}\left(b_{21}-a_{11}\right) \\
& \left.\times\left(a_{21}+b_{11}\right) \sin \alpha \cos ^{3} \alpha+\frac{3}{8}\left(\left(a_{21}+b_{11}\right)^{2}-\left(a_{11}-b_{21}\right)^{2}\right) \cos ^{4} \alpha\right] \rho, \\
= & r_{2}(\alpha, \rho)-\pi b_{01}\left(a_{11}+b_{21}\right)+\left[-\frac{\pi}{2}\left(2\left(a_{12}+b_{22}\right)+\left(a_{11}+b_{21}\right)\left(a_{21}-b_{11}\right)\right)\right. \\
& \left.+\frac{\pi}{2}\left(a_{11}+b_{21}\right)^{2}(\pi-\alpha)-\frac{\pi}{2}\left(a_{11}^{2}-b_{21}^{2}\right) \sin \alpha \cos \alpha-\frac{\pi}{2}\left(a_{11}+b_{21}\right)\left(a_{21}+b_{11}\right) \sin ^{2} \alpha\right] \rho . \\
r_{2}(-2 \pi+\alpha, \rho) &
\end{aligned}
$$

Proof. The expressions of $r_{1}(\theta, \rho)$ and $r_{2}(\theta, \rho)$ can be obtained using Corollary 2.4 or Proposition 2.6. The statement follows evaluating $r_{1}(\theta, \rho)$ and $r_{2}(\theta, \rho)$ in $\theta=\alpha$ and $\theta=-2 \pi+\alpha$.

Finally, we exemplify the algorithm of this section with the following proposition. Result below gives an example of a piecewise linear perturbation of the linear center that exhibits two limit cycles when $\alpha=\pi / 2$. 
Proposition 2.8. Consider, in $\Sigma_{\frac{\pi}{2}}^{ \pm}$, the system

$$
\begin{aligned}
& X^{+}:(\dot{x}, \dot{y})=\left(-y+(1-3 x) \varepsilon-8 \pi^{-1} x \varepsilon^{2}, x\right), \\
& X^{-}:(\dot{x}, \dot{y})=\left(-y+(1+x) \varepsilon-4 \varepsilon^{2}+\left(\beta_{1} x+\beta_{2}\right) \varepsilon^{3}, x\right),
\end{aligned}
$$

with $\beta_{1}=\left(3 \pi^{2}+8 \pi-48\right) /\left(3 \pi^{2}\right)$ and $\beta_{2}=\left(3 \pi^{2}+10 \pi-8\right) / \pi$. It has two hyperbolic limit cycles $\Gamma_{1}^{\varepsilon}$ and $\Gamma_{2}^{\varepsilon}$, for $\varepsilon$ small enough. Moreover, when $\varepsilon$ goes to 0 , the limit cycles $\Gamma_{1}^{\varepsilon}$ and $\Gamma_{2}^{\varepsilon}$ go to the circles $x^{2}+y^{2}=1$ and $x^{2}+y^{2}=4$, respectively.

Proof. We will prove that the first two Poincaré-Pontryagin-Melnikov functions vanish identically and the third one has exactly two simple zeros. The statement follows using Proposition 2.1.

Firstly, we write system (7) in the polar form. The vector fields $X^{ \pm}$write as

$$
(\dot{r}, \dot{\theta})= \begin{cases}\left(Y_{1}^{+}(r, \theta), Y_{2}^{+}(r, \theta)\right) & \text { if } \theta \in[0, \pi / 2), \\ \left(Y_{1}^{-}(r, \theta), Y_{2}^{-}(r, \theta)\right) & \text { if } \theta \in[\pi / 2,2 \pi),\end{cases}
$$

where

$$
\begin{aligned}
& Y_{1}^{+}(r, \theta)=\cos \theta(1-3 r \cos \theta) \varepsilon-8 \pi^{-1} r \cos ^{2} \theta \varepsilon^{2}, \\
& Y_{2}^{+}(r, \theta)=1-r^{-1} \sin \theta(1-3 r \cos \theta) \varepsilon+8 \pi^{-1} \sin \theta \cos \theta \varepsilon^{2}, \\
& Y_{1}^{-}(r, \theta)=\cos \theta(1+r \cos \theta) \varepsilon-4 \cos \theta \varepsilon^{2}+\cos \theta\left(\beta_{2}+\beta_{1} r \cos \theta\right) \varepsilon^{3}, \\
& Y_{2}^{-}(r, \theta)=1-r^{-1} \sin \theta(1+r \cos \theta) \varepsilon+4 r^{-1} \sin \theta \varepsilon^{2}-r^{-1} \sin \theta\left(\beta_{2}+\beta_{1} r \cos \theta\right) \varepsilon^{3} .
\end{aligned}
$$

System (8) can also be expressed as

$$
\begin{cases}W^{+}(r, \theta) & \text { if } \theta \in[0, \pi / 2) \\ W^{-}(r, \theta) & \text { if } \theta \in[\pi / 2,2 \pi)\end{cases}
$$

where

$$
\begin{aligned}
& W^{+}(r, \theta)=r d r-(1-3 r \cos \theta)(\sin \theta d r+r \cos \theta d \theta) \varepsilon+8 \pi^{-1} r \cos \theta(\sin \theta d r+r \cos \theta d \theta) \varepsilon^{2}, \\
& W^{-}(r, \theta)=r d r-(1+r \cos \theta)(\sin \theta d r+r \cos \theta d \theta) \varepsilon+4(\sin \theta d r+r \cos \theta d \theta) \varepsilon^{2}-\left(\beta_{2}+\beta_{1} r \cos \theta\right)(\sin \theta d r+r \cos \theta d \theta) \varepsilon^{3} .
\end{aligned}
$$

The analytic one-forms $\omega_{i}^{ \pm}$, defined in system (2), for system (8) are given by

$$
\begin{aligned}
& \omega_{1}^{+}(r, \theta)=-(1-3 r \cos \theta)(\sin \theta d r+r \cos \theta d \theta), \\
& \omega_{2}^{+}(r, \theta)=8 \pi^{-1} r \cos \theta(\sin \theta d r+r \cos \theta d \theta), \\
& \omega_{1}^{-}(r, \theta)=-(1+r \cos \theta)(\sin \theta d r+r \cos \theta d \theta), \\
& \omega_{2}^{-}(r, \theta)=4(\sin \theta d r+r \cos \theta d \theta), \\
& \omega_{3}^{-}(r, \theta)=-\left(\beta_{1} r \cos \theta+\beta_{2}\right)(\sin \theta d r+r \cos \theta d \theta),
\end{aligned}
$$

and $\omega_{3}^{+}(r, \theta)=0$.

Let $r^{ \pm}(\theta, \rho, \varepsilon)=r_{0}^{ \pm}(\theta, \rho)+r_{1}^{ \pm}(\theta, \rho) \varepsilon+r_{2}^{ \pm}(\theta, \rho) \varepsilon^{2}+r_{3}^{ \pm}(\theta, \rho) \varepsilon^{3}$ be the solution of the initial value problem $W^{ \pm}(r, \theta)=$ $0, r^{ \pm}(0, \rho, \varepsilon)=\rho$. It is easy to check that $r_{0}^{ \pm}(\theta, \rho) \equiv \rho$ and $r_{i}^{ \pm}(0, \rho)=0$.

Now we start with the computation of the first order term of the difference map. We use Lemma 2.2 in order to obtain a decomposition of the one-forms $\Omega_{1}^{ \pm}$defined in Theorem 2.3. If we write them as $-\Omega_{1}^{ \pm}(r, \theta)=-\omega_{1}^{ \pm}=\alpha_{1}^{ \pm}(r, \theta) d r+\beta_{1}^{ \pm}(r, \theta) d \theta$, then the functions $F_{1}^{ \pm}(r), S_{1}^{ \pm}(r, \theta)$ and $h_{1}^{ \pm}(r, \theta)$ are obtained from the decomposition of Lemma 2.2 of the one-forms $-\Omega_{1}^{ \pm}$. Consequently,

$$
\begin{aligned}
F_{1}^{ \pm}(r) & =\frac{1}{2 \pi} \int_{0}^{2 \pi} \beta_{1}^{ \pm}(r, \psi) d \psi, \quad h_{1}^{ \pm}(r, \theta)=\frac{1}{r}\left(\alpha_{1}^{ \pm}(r, \theta)-D_{1}\left(S_{1}^{ \pm}\right)(r, \theta)\right) \\
S_{1}^{ \pm}(r, \theta) & =\int_{0}^{\theta} \beta_{1}^{ \pm}(r, \psi) d \psi-F_{1}^{ \pm}(r) \theta .
\end{aligned}
$$

Thus, after some direct calculations, we obtain the following expressions:

$$
\begin{array}{ll}
F_{1}^{+}(r)=-3 r^{2} / 2, & S_{1}^{+}(r, \theta)=r \sin \theta-3 r^{2} \sin (2 \theta) / 4, \quad h_{1}^{+}(r, \theta)=0, \\
F_{1}^{-}(r)=r^{2} / 2, & S_{1}^{-}(r, \theta)=r \sin \theta+r^{2} \sin (2 \theta) / 4, \quad h_{1}^{-}(r, \theta)=0 .
\end{array}
$$

In order to obtain $r_{1}^{ \pm}(\theta, \rho)$, from Corollary 2.4, we need to solve the equation $\rho r_{1}^{ \pm}(\theta, \rho)=F_{1}^{ \pm}(\rho) \theta+S_{1}^{ \pm}(\rho, \theta)-S_{1}^{ \pm}(\rho, 0)$, which simplifies to

$$
\begin{aligned}
& \rho r_{1}^{+}(\theta, \rho)=-3(\theta+\cos \theta \sin \theta) \rho^{2} / 2+\rho \sin \theta, \\
& \rho r_{1}^{-}(\theta, \rho)=(\theta+\cos \theta \sin \theta) \rho^{2} / 2+\rho \sin \theta .
\end{aligned}
$$

Therefore, $r_{1}^{+}(\theta, \rho)=-3(\theta+\cos \theta \sin \theta) \rho / 2+\sin \theta$ and $r_{1}^{-}(\theta, \rho)=(\theta+\cos \theta \sin \theta) \rho / 2+\sin \theta$. Consequently $r_{1}^{+}(\pi / 2, \rho)=$ $r_{1}^{-}(-3 \pi / 2, \rho)=-3 \pi \rho / 4+1$ and the first coefficient of the difference map $M_{1}(\rho) \equiv 0$. 
For the second order term we use again Lemma 2.2 to decompose the one-forms

$$
\begin{aligned}
& -\Omega_{2}^{+}=-\omega_{1}^{+} h_{1}^{+}-\omega_{2}^{+}=-\omega_{2}^{+}=-8 \pi^{-1} r \cos \theta \sin \theta d r-8 \pi^{-1} r^{2} \cos ^{2} \theta d \theta, \\
& -\Omega_{2}^{-}=-\omega_{1}^{-} h_{1}^{-}-\omega_{2}^{-}=-\omega_{2}^{-}=-4 \sin \theta d r-4 r \cos \theta d \theta .
\end{aligned}
$$

We write them as $-\Omega_{2}^{ \pm}(r, \theta)=\alpha_{2}^{ \pm}(r, \theta) d r+\beta_{2}^{ \pm}(r, \theta) d \theta$. The functions $F_{2}^{ \pm}, S_{2}^{ \pm}$and $h_{2}^{ \pm}$are given by

$$
\begin{aligned}
F_{2}^{+}(r) & =\frac{1}{2 \pi} \int_{0}^{2 \pi} \beta_{2}^{+}(r, \psi) d \psi=\frac{1}{2 \pi} \int_{0}^{2 \pi}-\frac{8 r^{2} \cos ^{2} \psi}{\pi} d \psi=-\frac{4}{\pi} r^{2}, \\
S_{2}^{+}(r, \theta) & =\int_{0}^{\theta} \beta_{2}^{+}(r, \psi) d \psi-F_{2}^{+}(r) \theta=\int_{0}^{\theta}-\frac{8 r^{2} \cos ^{2} \psi}{\pi} d \psi+\frac{4}{\pi} r^{2} \theta=-\frac{2}{\pi} r^{2} \sin (2 \theta), \\
h_{2}^{+}(r, \theta) & =r^{-1}\left(\alpha_{2}^{+}(r, \theta)-D_{1}\left(S_{2}^{+}\right)(r, \theta)\right)=r^{-1}\left(-8 \pi^{-1} r \cos \theta \sin \theta\right)+4 \pi^{-1} r \sin (2 \theta)=0, \\
F_{2}^{-}(r) & =\frac{1}{2 \pi} \int_{0}^{2 \pi} \beta_{2}^{-}(r, \psi) d \psi=\frac{1}{2 \pi} \int_{0}^{2 \pi}-4 r \cos \psi d \psi=0, \\
S_{2}^{-}(r, \theta) & =\int_{0}^{\theta} \beta_{2}^{-}(r, \psi) d \psi-F_{2}^{-}(r) \theta=\int_{0}^{\theta}-4 r \cos \psi d \psi=-4 r \sin \theta, \\
h_{2}^{-}(r, \theta) & =r^{-1}\left(\alpha_{2}^{-}(r, \theta)-D_{1}\left(S_{2}^{-}\right)(r, \theta)\right)=r^{-1}(-4 \sin \theta+4 \sin \theta)=0 .
\end{aligned}
$$

In order to obtain $r_{2}^{ \pm}(\theta, \rho)$, again from Corollary 2.4 , we need to solve the equations $\rho r_{2}^{ \pm}(\theta, \rho)=\mathcal{F}_{2}^{ \pm}\left(\theta, \rho, r_{1}\right)$, where

$$
\mathcal{F}_{2}^{ \pm}=F_{2}^{ \pm}(\rho) \theta+\left.\left(S_{2}^{ \pm}(\rho, \psi)+\frac{\partial S_{1}^{ \pm}}{\partial r}(\rho, \psi) r_{1}^{ \pm}(\psi, \rho)\right)\right|_{\psi=0} ^{\psi=\theta}-\frac{1}{2} r_{1}^{ \pm}(\theta, \rho)^{2}+\left(F_{1}^{ \pm}\right)^{\prime}(\rho) \int_{0}^{\theta} r_{1}^{ \pm}(\psi, \rho) d \psi .
$$

From these equations we obtain the following expressions for the functions $r_{2}^{ \pm}(\theta, \rho)$

$$
\begin{aligned}
& r_{2}^{+}(\theta, \rho)=\left[\frac{9}{4}-\frac{4 \theta}{\pi}+\frac{9 \theta^{2}}{8}+\frac{9}{8}\left(1-3 \cos ^{2} \theta\right) \cos ^{2} \theta+\left(\frac{9 \theta}{4}-\frac{4}{\pi}\right) \cos \theta \sin \theta\right] \rho+3 \cos ^{3} \theta-3+\frac{\sin ^{2} \theta}{2 \rho}, \\
& r_{2}^{-}(\theta, \rho)=\left[\frac{1}{4}+\frac{\theta^{2}}{8}+\frac{1}{8}\left(1-3 \cos ^{2} \theta\right) \cos ^{2} \theta+\frac{\theta}{4} \cos \theta \sin \theta\right] \rho-\cos ^{3} \theta-4 \sin \theta+1+\frac{\sin ^{2} \theta}{2 \rho}
\end{aligned}
$$

Consequently $r_{2}^{+}(\pi / 2, \rho)=r_{2}^{-}(-3 \pi / 2, \rho)=\left(9 \pi^{2}+8\right) \rho / 32-3+1 /(2 \rho)$ and $M_{2}(\rho) \equiv 0$.

We finish with the third order term. We use again Lemma 2.2 to decompose the one-forms $-\Omega_{3}^{+}=-\omega_{1}^{+} h_{2}^{+}-\omega_{2}^{+} h_{1}^{+}-\omega_{3}^{+}=-\omega_{3}^{+}=0$ and $-\Omega_{3}^{-}=-\omega_{1}^{-} h_{2}^{-}-\omega_{2}^{-} h_{1}^{-}-\omega_{3}^{-}=-\omega_{3}^{-}$. The expression for $\omega_{3}^{-}$can be found above. Similarly as in the first and second order, one can compute the functions $F_{3}^{ \pm}, S_{3}^{ \pm}$and $h_{3}^{ \pm}$that appear in the decomposition of Lemma 2.2 of the one-forms $-\Omega_{3}^{ \pm}$. They are given by $F_{3}^{-}(r)=\beta_{1} r^{2} / 2, S_{3}^{-}(r, \theta)=\beta_{2} r \sin \theta+\beta_{1} r^{2} \sin (2 \theta)$, and $h_{3}^{ \pm}(r, \theta)=F_{3}^{+}(r)=S_{3}^{+}(r, \theta)=0$.

Using again Corollary 2.4, we can obtain the functions $r_{3}^{ \pm}(\theta, \rho)$. For simplicity, we omit the expressions of them. Consequently,

$$
\begin{aligned}
r_{3}^{+}(\pi / 2, \rho) & =-3\left(3 \pi^{4}+44 \pi^{2}-512\right) \rho /(128 \pi)+\left(9 \pi^{2}-32\right) /(4 \pi)-3 \pi /(8 \rho), \\
r_{3}^{-}(-3 \pi / 2, \rho) & =-\left(9 \pi^{4}+132 \pi^{2}+256 \pi-1536\right) /(128 \pi) \rho+\left(9 \pi^{2}+24 \pi-32\right) /(4 \pi)-(3 \pi+32) /(8 \rho),
\end{aligned}
$$

and $M_{3}(\rho)=2(\rho-1)(\rho-2) / \rho$. The proof finishes because $\rho=1$ and $\rho=2$ are two simple zeros of $M_{3}(\rho)$ and $M_{1}(\rho) \equiv M_{2}(\rho) \equiv 0$.

\section{Higher order perturbations for $\Sigma_{\alpha}$-piecewise linear systems}

In this section we study the number of limit cycles that appear from piecewise linear perturbations up to order six of a linear center when the separation line has only a breaking point. The next result provides the maximum number of zeros of the Poincaré-Pontryagin-Melnikov function of order $N$ for the first values of $N$.

Proposition 3.1. For system (1), the maximum number of zeros of the corresponding function $M_{N}(\rho)$ is $1,2,2,3,4,5$ when $N=1, \ldots, 6$.

Proof. Let $P_{i}^{ \pm}(x, y)=a_{0 i}^{ \pm}+a_{1 i}^{ \pm} x+a_{2 i}^{ \pm} y$ and $Q_{i}^{ \pm}(x, y)=b_{0 i}^{ \pm}+b_{1 i}^{ \pm} x+b_{2 i}^{ \pm} y$ be the polynomials defined in (1). By Propositions 2.5 and 2.6 and Corollary 2.7, substituting the parameters $a_{0 i}, a_{1 i}, a_{2 i}, b_{0 i}, b_{1 i}$ and $b_{2 i}$ by the respective $a_{0 i}^{ \pm}, a_{1 i}^{ \pm}, a_{2 i}^{ \pm}, b_{0 i}^{ \pm}, b_{1 i}^{ \pm}$and $b_{2 i}^{ \pm}$, we get $r_{i}^{+}(\alpha, \rho)$ and $r_{i}^{-}(-2 \pi+\alpha, \rho)$, for $i=1, \ldots, 6$. So, from Proposition 2.1 we have that

$$
\begin{aligned}
M_{1}(\rho)=\widehat{M}_{1}(\rho)= & \left(a_{01}^{+}-a_{01}^{-}\right) \sin \alpha+\left(b_{01}^{+}-b_{01}^{-}\right)(1-\cos \alpha) \\
& +\frac{1}{2}\left[\left(a_{11}^{+}-a_{11}^{-}\right)(\alpha+\sin \alpha \cos \alpha)+\left(b_{21}^{+}-b_{21}^{-}\right)(\alpha-\sin \alpha \cos \alpha)\right. \\
& \left.+\left(a_{21}^{+}-a_{21}^{-}+b_{11}^{+}-b_{11}^{-}\right) \sin ^{2} \alpha+2 \pi\left(a_{11}^{-}+b_{21}^{-}\right)\right] \rho=C_{0,1}+C_{1,1} \rho .
\end{aligned}
$$

In general, for $2 \leq i \leq 6$, the Poincaré-Pontryagin-Melnikov functions write as

$$
\widehat{M}_{i}(\rho)=\sum_{j=1-i}^{1} C_{j, i}\left(\lambda_{i}\right) \rho^{j}
$$


where $C_{j, i}(\lambda)$ are polynomials of degree $i$ in the variables

$$
\lambda=\left(a_{01}^{ \pm}, a_{11}^{ \pm}, a_{21}^{ \pm}, b_{01}^{ \pm}, b_{11}^{ \pm}, b_{21}^{ \pm}, \ldots, a_{0 i}^{ \pm}, a_{1 i}^{ \pm}, a_{2 i}^{ \pm}, b_{0 i}^{ \pm}, b_{1 i}^{ \pm}, b_{2 i}^{ \pm}\right) .
$$

We omit the explicit expressions of the polynomials $C_{j, i}\left(\lambda_{i}\right)$ because of the size of them. We have that $M_{1}(\rho) \equiv 0$ if and only if $C_{0,1}=C_{1,1}=0$, that is

$$
\begin{aligned}
a_{01}^{-}= & (\sin \alpha)^{-1}\left(a_{01}^{+} \sin \alpha+\left(b_{01}^{+}-b_{01}^{-}\right)(1-\cos \alpha)\right), \\
a_{11}^{-}= & (\alpha-2 \pi+\sin \alpha \cos \alpha)^{-1}\left(a_{11}^{+}(\alpha+\sin \alpha \cos \alpha)+\left(b_{21}^{+}-b_{21}^{-}\right)(\alpha-\sin \alpha \cos \alpha)\right. \\
& \left.+\left(a_{21}^{+}-a_{21}^{-}+b_{11}^{+}-b_{11}^{-}\right) \sin ^{2} \alpha+2 \pi b_{21}^{-}\right) .
\end{aligned}
$$

Then, the polynomial $C_{-1,2}$ corresponding to function $\widehat{M}_{2}(\rho)$ becomes

$$
C_{-1,2}=\left(b_{01}^{-}-b_{01}^{+}\right)\left(a_{01}^{+} \sin \alpha+b_{01}^{+}(1-\cos \alpha)\right) .
$$

If $b_{01}^{-} \neq b_{01}^{+}$, then $C_{-1,2} \equiv 0$ if and only if

$$
a_{01}^{+}=\frac{(\cos \alpha-1) b_{01}^{+}}{\sin \alpha}
$$

Imposing that the parameters $a_{01}^{-}, a_{11}^{-}$and $a_{01}^{+}$are like in (9) and (10) we obtain that $C_{-2,3}=C_{-3,4}=C_{-4,5}=C_{-5,6}=0$. This shows that the degrees of the functions $\widehat{M}_{3}(\rho), \widehat{M}_{4}(\rho), \widehat{M}_{5}(\rho)$, and $\widehat{M}_{6}(\rho)$ are at most $2,3,4$, and 5 , respectively. Therefore, when $N=1, \ldots, 6$, the maximum number of zeros of $\widehat{M}_{N}(\rho)$ is $1,2,2,3,4,5$, respectively. The proof concludes because the zeros of $M_{N}(\rho)$ and $\widehat{M}_{N}(\rho)$ agree.

The result below shows the existence of an example of a piecewise linear perturbation of the linear center that exhibits four limit cycles when the separation line is nonregular.

Proposition 3.2. For every $\alpha \in(0, \pi)$, there exist real numbers $a_{01}^{+}, a_{11}^{+}, a_{21}^{+}, a_{02}^{+}, a_{22}^{+}, a_{03}^{+}, a_{04}^{+}, a_{0 i}^{-}$, and $a_{1 i}^{-}$, for $i=1, \ldots, 5$, such that the system

$$
\begin{aligned}
& X^{+}:\left\{\begin{array}{l}
\dot{x}=-y+\left(a_{01}^{+}+a_{11}^{+} x+a_{21}^{+} y\right) \varepsilon+\left(a_{02}^{+}+a_{22}^{+} y\right) \varepsilon^{2} \\
+a_{03}^{+} \varepsilon^{3}+a_{04}^{+} \varepsilon^{4} \\
\dot{y}=x+2 \varepsilon
\end{array}\right. \\
& X^{-}:\left\{\begin{array}{l}
\dot{x}=-y+\sum_{i=1}^{5} \varepsilon^{i}\left(a_{0 i}^{-}+a_{1 i}^{-} x\right), \\
\dot{y}=x+\varepsilon,
\end{array}\right.
\end{aligned}
$$

has 4 hyperbolic limit cycles, for $\varepsilon$ small enough.

Proof. From the procedure described in Section 2, Proposition 2.6, and Corollary 2.7 we get $r_{i}^{+}(\alpha, \rho)$ and $r_{i}^{-}(-2 \pi+\alpha, \rho)$, for $i=1, \ldots, 5$. So, from Proposition 2.1, we have that the Poincaré-Pontryagin-Melnikov functions, for $i=1, \ldots, 5$, write as

$$
\widehat{M}_{i}(\rho)=\sum_{j=1-i}^{1} C_{j, i}(\lambda) \rho^{j},
$$

where $C_{j, i}(\lambda)$ are given polynomials of degree $i$ in the variables

$$
\lambda=\left(a_{01}^{ \pm}, a_{02}^{ \pm}, a_{03}^{ \pm}, a_{04}^{ \pm}, a_{05}^{-}, a_{11}^{ \pm}, a_{12}^{-}, a_{13}^{-}, a_{14}^{-}, a_{15}^{-}, a_{21}^{+}, a_{22}^{+}\right) .
$$

We start with a short scheme of the proof. We prove that, for every $\alpha$, there are parameters $\lambda$ such that $M_{1}(\rho) \equiv M_{2}(\rho) \equiv M_{3}(\rho) \equiv$ $M_{4}(\rho) \equiv 0$ and

$$
M_{5}(\rho)=G_{0} \rho^{-3}+G_{1} \rho^{-2}+G_{2} \rho^{-1}+G_{3}+\rho
$$

where $G_{i}$ for $i=0, \ldots, 3$ are arbitrary constants. Therefore, $M_{5}(\rho)$ can have 4 simple zeros. For that we consider a sequence of systems of equations, $E_{k}, k=1, \ldots, 7$, involving the coefficients of $\widehat{M}_{1}, \ldots, \widehat{M}_{5}$ with respect to $\rho$, which are denoted by $C_{j, i}(\lambda)$. The systems $E_{k}$ are linear with respect to a specific collection of variables, $\lambda_{k}$, that are a subset of the full parameter $\lambda$. More precisely we follow the next steps. First we start considering the system of equations, $E_{1}(\alpha)$, defined by the coefficients of the function $\widehat{M}_{1}(\rho)$. Solving $E_{1}(\alpha)$ with respect to $\lambda_{1}$ we vanish identically $\widehat{M}_{1}(\rho)$. The second system, $E_{2}$, is defined by the coefficient of lower degree of $\widehat{M}_{2}$. As in the proof of Proposition 3.1, when this coefficient vanishes all the coefficients of lower degree of $\widehat{M}_{3}, \widehat{M}_{4}$, and $\widehat{M}_{5}$ also vanish. For each $k=3$, .., 7, we consider the system $E_{k}(\alpha)$ formed by the polynomials $C_{j, i}(\lambda)$ such that $j+i=k-1$. Step by step, we show that there are variables $\lambda_{k}$ such that, with respect to them, the system $E_{k}(\alpha)$ is linear and it can be uniquely solved. The determinant of the corresponding matrix $A_{k}(\alpha)$ of the coefficients of $E_{k}(\alpha)$ with respect to $\lambda_{k}$ is nonzero, for all $\alpha \in(0, \pi)$, so that there exists a unique solution such that $M_{5}$ has indeterminate coefficients. 
Now we continue with the full proof. For the vector fields $X^{+}$and $X^{-}$, using the method of Section 2 the functions $F_{i}^{ \pm}, h_{i}^{ \pm}$, and $S_{i}^{ \pm}$in Proposition 2.5 write as

$$
\begin{aligned}
& F_{1}^{+}(r)=\frac{1}{2} a_{11}^{+} r^{2}, \\
& h_{1}^{+}(r, \theta)=\frac{1}{2} a_{21}^{+} \text {, } \\
& F_{2}^{+}(r)=\frac{1}{4} a_{11}^{+} a_{21}^{+} r^{2}, \\
& h_{2}^{+}(r, \theta)=\frac{1}{4}\left[\left(a_{21}^{+}\right)^{2}+2 a_{22}^{+}\right] \text {, } \\
& F_{3}^{+}(r)=\frac{1}{8} a_{11}^{+}\left[\left(a_{21}^{+}\right)^{2}+2 a_{22}^{+}\right] r^{2}, \\
& h_{3}^{+}(r, \theta)=\frac{1}{8}\left[\left(a_{21}^{+}\right)^{3}+4 a_{21}^{+} a_{22}^{+}\right] \text {, } \\
& F_{4}^{+}(r)=\frac{1}{16} a_{11}^{+}\left[\left(a_{21}^{+}\right)^{3}+4 a_{21}^{+} a_{22}^{+}\right] r^{2}, \\
& h_{4}^{+}(r, \theta)=\frac{1}{16}\left[\left(a_{21}^{+}\right)^{4}+6\left(a_{21}^{+}\right)^{2} a_{22}^{+}+4\left(a_{22}^{+}\right)^{2}\right] \text {, } \\
& F_{5}^{+}(r)=\frac{1}{32} a_{11}^{+}\left[\left(a_{21}^{+}\right)^{4}+6\left(a_{21}^{+}\right)^{2} a_{22}^{+}+4\left(a_{22}^{+}\right)^{2}\right] r^{2}, h_{5}^{+}(r, \theta)=\frac{1}{32}\left[\left(a_{21}^{+}\right)^{5}+8\left(a_{21}^{+}\right)^{3} a_{22}^{+}+12 a_{21}^{+}\left(a_{22}^{+}\right)^{2}\right] \text {, } \\
& F_{i}^{-}(r)=\frac{1}{2} a_{1 i}^{-} r^{2}, \quad \text { for } i=1, \ldots, 5, \quad h_{i}^{-}(r, \theta)=0, \quad \text { for } i=1, \ldots, 5 \text {, } \\
& S_{1}^{-}(r, \theta)=\frac{1}{2}\left[2 a_{01}^{-}+a_{11}^{-} r \cos \theta\right] r \sin \theta-r \cos \theta, \\
& S_{i}^{-}(r, \theta)=\frac{1}{2}\left[2 a_{0 i}^{-}+a_{1 i}^{-} r \cos \theta\right] r \sin \theta, \quad \text { for } i=2, \ldots, 5 \text {, } \\
& S_{1}^{+}(r, \theta)=\frac{1}{4} r\left[4 a_{01}^{+} \sin \theta-8 \cos \theta+2 a_{11}^{+} r \sin \theta \cos \theta+a_{21}^{+} r\left(1-2 \cos ^{2} \theta\right)\right], \\
& S_{2}^{+}(r, \theta)=\frac{1}{8} r\left[\left(4\left(a_{01}^{+} a_{21}^{+}+2 a_{02}^{+}\right)+2 a_{11}^{+} a_{21}^{+} r \cos \theta\right) \sin \theta+\left(\left(a_{21}^{+}\right)^{2}+a_{22}^{+}\right) r\left(1-2 \cos ^{2} \theta\right)-8 a_{21}^{+} \cos \theta\right] \text {, } \\
& S_{3}^{+}(r, \theta)=\frac{1}{16} r\left[4\left(a_{01}^{+}\left(a_{21}^{+}\right)^{2}+2 a_{01}^{+} a_{22}^{+}+2 a_{21}^{+} a_{02}^{+}+4 a_{03}^{+}\right) \sin \theta+2\left(a_{11}^{+}\left(a_{21}^{+}\right)^{2}+2 a_{11}^{+} a_{22}^{+}\right) r \sin \theta \cos \theta\right. \\
& \left.+a_{21}^{+}\left(\left(a_{21}^{+}\right)^{2}+4 a_{22}^{+}\right) r\left(1-2 \cos ^{2} \theta\right)-8\left(\left(a_{21}^{+}\right)^{2}+2 a_{22}^{+}\right) \cos \theta\right] \text {, } \\
& S_{4}^{+}(r, \theta)=\frac{1}{32} r\left[4\left(a_{01}^{+}\left(a_{21}^{+}\right)^{3}+4 a_{01}^{+} a_{21}^{+} a_{22}^{+}+2\left(a_{21}^{+}\right)^{2} a_{02}^{+}+4 a_{02}^{+} a_{22}^{+}+4 a_{21}^{+} a_{03}^{+}+8 a_{04}^{+}\right) \sin \theta\right. \\
& -8\left(\left(a_{21}^{+}\right)^{3}+4 a_{21}^{+} a_{22}^{+}\right) \cos \theta+2\left(a_{11}^{+}\left(a_{21}^{+}\right)^{3}+4 a_{11}^{+} a_{21}^{+} a_{22}^{+}\right) r \sin \theta \cos \theta \\
& \left.+\left(\left(a_{21}^{+}\right)^{4}+6\left(a_{21}^{+}\right)^{2} a_{22}^{+}+4\left(a_{22}^{+}\right)^{2}\right) r\left(1-2 \cos ^{2} \theta\right)\right], \\
& S_{5}^{+}(r, \theta)=\frac{1}{64} r\left[4 \left(a_{01}^{+}\left(a_{21}^{+}\right)^{4}+4 a_{01}^{+}\left(a_{22}^{+}\right)^{2}+2\left(a_{21}^{+}\right)^{3} a_{02}^{+}+4\left(a_{21}^{+}\right)^{2} a_{03}^{+}+8 a_{03}^{+} a_{22}^{+}+8 a_{04}^{+} a_{21}^{+}+6 a_{01}^{+}\left(a_{21}^{+}\right)^{2} a_{22}^{+}\right.\right. \\
& \left.+8 a_{02}^{+} a_{21}^{+} a_{22}^{+}\right) \sin \theta-8\left(\left(a_{21}^{+}\right)^{4}+6\left(a_{21}^{+}\right)^{2} a_{22}^{+}+4\left(a_{22}^{+}\right)^{2}\right) \cos \theta+2\left(a_{11}^{+}\left(a_{21}^{+}\right)^{4}+6 a_{11}^{+}\left(a_{21}^{+}\right)^{2} a_{22}^{+}\right. \\
& \left.\left.+4 a_{11}^{+}\left(a_{22}^{+}\right)^{2}+\left(\left(a_{21}^{+}\right)^{5}+8\left(a_{21}^{+}\right)^{3} a_{22}^{+}+12 a_{21}^{+}\left(a_{22}^{+}\right)^{2}\right) r\left(1-2 \cos ^{2} \theta\right)\right) r \sin \theta \cos \theta\right] \text {. }
\end{aligned}
$$

The first step starts defining $E_{1}(\alpha)$ and $\lambda_{1}$. We have that the coefficients $C_{0,1}$ and $C_{1,1}$ of the function $\widehat{M}_{1}(\rho)$ are linear with respect to variables $a_{01}^{-}, a_{01}^{+}, a_{11}^{-}, a_{11}^{+}$, and $a_{21}^{+}$. Consequently $E_{1}(\alpha)=\left\{C_{0,1}=0, C_{1,1}=0\right\}$ and $\lambda_{1}=\left(a_{01}^{-}, a_{11}^{-}\right)$, where

$$
\begin{aligned}
& C_{0,1}=\left(a_{01}^{+}-a_{01}^{-}\right) \sin \alpha+1-\cos \alpha, \\
& C_{1,1}=\frac{1}{2}\left[\left(a_{11}^{+}-a_{11}^{-}\right)(\alpha+\sin \alpha \cos \alpha)+a_{21}^{+} \sin ^{2} \alpha+2 a_{11}^{-} \pi\right] .
\end{aligned}
$$

Let $A_{1}(\alpha)$ be the matrix of the coefficients of $E_{1}(\alpha)$ with respect to $\lambda_{1}$. We have that $\operatorname{det} A_{1}(\alpha)=\sin \alpha(\cos \alpha \sin \alpha-2 \pi+\alpha) / 2 \neq 0$, for all $\alpha \in(0, \pi)$, so that there exists a unique solution of system $E_{1}(\alpha)$. That is

$$
a_{01}^{-}=\frac{1+a_{01}^{+} \sin \alpha-\cos \alpha}{\sin \alpha}, \quad a_{11}^{-}=\frac{a_{11}^{+} \alpha+a_{21}^{+} \sin ^{2} \alpha+a_{11}^{+} \sin \alpha \cos \alpha}{\alpha-2 \pi+\sin \alpha \cos \alpha} .
$$

Then we obtain that $\widehat{M}_{1}(\rho) \equiv 0$.

The second step continues considering the coefficients of $\widehat{M}_{2}(\rho)$ :

$$
\begin{aligned}
C_{-1,2} & =-a_{01}^{+} \sin \alpha+2(\cos \alpha-1), \\
C_{0,2} & =-a_{02}^{-} \sin \alpha+U_{2}\left(a_{01}^{+}, a_{02}^{+}, a_{11}^{+}, a_{21}^{+}\right), \\
C_{1,2} & =\frac{1}{2} a_{12}^{-}(2 \pi-\alpha-\sin \alpha \cos \alpha)+V_{2}\left(a_{11}^{+}, a_{21}^{+}, a_{22}^{+}\right),
\end{aligned}
$$

where $U_{2}$ and $V_{2}$ are polynomials of degree two in the respective parameters. Note that $C_{-1,2}$ is linear with respect to the variable $a_{01}^{+}$. Consequently $E_{2}(\alpha)=\left\{C_{-1,2}=0\right\}$ and $\lambda_{2}=\left(a_{01}^{+}\right)$. If we denote by $A_{2}$ the matrix of the coefficients of $U_{2}$ with respect to $\lambda_{2}$, in fact $A_{2}$ is a real number, we see that $\operatorname{det} A_{2}(\alpha)=-\sin \alpha \neq 0$, for all $\alpha \in(0, \pi)$. So the solution of $E_{2}(\alpha)$ with respect to $\lambda_{2}$ is

$$
a_{01}^{+}=2 \frac{\cos \alpha-1}{\sin \alpha} \text {. }
$$

As we have mentioned before, this implies that the coefficients $C_{-2,3}, C_{-3,4}$, and $C_{-4,5}$ also vanish. 
For the last steps we omit, due to the size of them, the explicit expression of the polynomials $C_{j, i}(\lambda)$, for $i=3,4,5$. In what follows we only explicit the systems $E_{k}$ and the variables $\lambda_{k}$ aforementioned:

$$
\begin{array}{ll}
E_{3}(\alpha)=\left\{C_{0,2}=0, C_{-1,3}=0, C_{-2,4}=0, C_{-3,5}=G_{0}\right\}, & \lambda_{3}=\left(a_{02}^{-}, a_{02}^{+}, a_{21}^{+}, a_{11}^{+}\right), \\
E_{4}(\alpha)=\left\{C_{1,2}=0, C_{0,3}=0, C_{-1,4}=0, C_{-2,5}=G_{1}\right\}, & \lambda_{4}=\left(a_{12}^{-}, a_{22}^{+}, a_{03}^{-}, a_{03}^{+}\right), \\
E_{5}(\alpha)=\left\{C_{1,3}=0, C_{0,4}=0, C_{-1,5}=G_{2}\right\}, & \lambda_{5}=\left(a_{13}^{-}, a_{04}^{-}, a_{04}^{+}\right), \\
E_{6}(\alpha)=\left\{C_{1,4}=0, C_{0,5}=G_{3}\right\}, & \lambda_{6}=\left(a_{14}^{-}, a_{05}^{-}\right), \\
E_{7}(\alpha)=\left\{C_{1,5}=1\right\}, & \lambda_{7}=\left(a_{15}^{-}\right) .
\end{array}
$$

The determinants of the respective matrices $A_{k}(\alpha)$, for $k=3, \ldots, 7$ are

$$
\begin{aligned}
& \operatorname{det} A_{3}(\alpha)=\frac{8(\cos \alpha-1)^{3}\left(7 \cos ^{2} \alpha-24 \pi \sin \alpha+15 \alpha \sin \alpha+16 \cos \alpha-23\right)}{9(\cos \alpha+1)(\cos \alpha \sin \alpha-2 \pi+\alpha)}, \\
& \operatorname{det} A_{4}(\alpha)=-(\cos \alpha-1)^{2}(2 \cos \alpha \sin \alpha+6 \pi \cos \alpha-3 \alpha \cos \alpha+4 \sin \alpha+6 \pi-3 \alpha) / 3, \\
& \operatorname{det} A_{5}(\alpha)=-\sin ^{2} \alpha(\cos \alpha \sin \alpha-2 \pi+\alpha) / 2, \\
& \operatorname{det} A_{6}(\alpha)=-\left(\cos ^{3} \alpha-2 \pi \sin \alpha+\alpha \sin \alpha+\cos \alpha\right) / 2, \\
& \operatorname{det} A_{7}(\alpha)=-(\cos \alpha \sin \alpha+2 \pi-\alpha) / 2
\end{aligned}
$$

Simple calculations show that all of above determinants are nonzero for all $\alpha \in(0, \pi)$. So that there exists a unique solution of each system $E_{k}(\alpha)$ with respect to variables $\lambda_{k}$ such that $\widehat{M}_{i} \equiv 0$ for $i=1, \ldots, 4$ and the coefficients of $\widehat{M}_{5}$ are $G_{k}$ for $k=0, \ldots, 3$.

The proof finishes from the Implicit Function Theorem that guarantees that each simple zero, $\rho_{j}$, of $M_{i}$, or equivalently $\widehat{M}_{i}$, gets a limit cycle for system (11). In fact the limit cycles bifurcate from the circles $x^{2}+y^{2}=\rho_{j}^{2}$, for $\varepsilon$ small enough.

We note that, usually when we study a Poincaré-Pontryagin-Melnikov function of some given order, we have proved first that the previous vanish identically. That is, for each order we solve the system of equations defined by all the coefficients of the corresponding function. In the above proof we have used another approach to prove that the fifth function has indeterminate coefficients and the previous are identically zero. In each step the system is defined from the coefficients of lower degree monomials of all the functions simultaneously.

Remark 3.3. It is not difficult to check that when $\alpha$ goes to $\pi$, the coefficient $C_{-3,5}$ of the function $\widehat{M}_{5}(\rho)$ goes to infinity. Once $C_{-3,5}$ is defined by the product of the radius of the four limit cycles, at least one radius goes to infinity. This means that when $\alpha$ goes to $\pi$, at least one limit cycle goes to infinity. When $\alpha$ goes to 0 all of the coefficients of the functions $\widehat{M}_{i}(\rho)$, for $i=1, \ldots, 5$, except the one of maximal degree, go to zero. Therefore all of the limit cycles go to origin. In particular, when $\alpha$ goes to 0 the system becomes a purely linear system, for which it is known that no limit cycles exist.

The proof of the first part of Theorem 1.1 follows directly from Propositions 3.1 and 3.2. The second part is proved in the next section.

\section{An explicit example with five limit cycles}

In this section first we provide an example of a concrete system (1), with $\alpha=\pi / 2$, exhibiting five limit cycles up to a perturbation of order 6 . Second we give the values where two of the limit cycles, that appear from the level curves of the center, disappear in a semistable limit cycle bifurcation. Similar systems can be obtained for other values of $\alpha$, like $\alpha=\pi / 3$ or $\alpha=\pi / 4$, but the expressions to manipulate are too big to provide the same result for every $\alpha$. In fact this can be used as an illustration of Proposition 3.2 where another type of piecewise linear system has four limit cycles up to a perturbation of order 5 , but for any $\alpha$.

Proposition 4.1. Consider, in $\Sigma_{\frac{\pi}{2}}^{ \pm}$, the system

$$
X^{ \pm}:(\dot{x}, \dot{y})=\left(-y+\sum_{i=1}^{6} \varepsilon^{i}\left(a_{0 i}^{ \pm}+a_{1 i}^{ \pm} x\right), x+\sum_{i=1}^{6} \varepsilon^{i}\left(b_{0 i}^{ \pm}+b_{1 i}^{ \pm} x\right)\right),
$$

with $a_{03}^{-}=a_{04}^{-}=a_{06}^{-}=a_{12}^{-}=a_{13}^{-}=a_{14}^{-}=a_{15}^{-}=b_{01}^{-}=b_{02}^{-}=b_{03}^{-}=b_{05}^{-}=b_{06}^{-}=b_{12}^{-}=b_{13}^{-}=b_{14}^{-}=b_{15}^{-}=b_{16}^{-}=a_{02}^{+}=a_{06}^{+}=a_{14}^{+}=$ $a_{15}^{+}=a_{16}^{+}=b_{01}^{+}=b_{05}^{+}=b_{06}^{+}=b_{11}^{+}=b_{16}^{+}=0, a_{01}^{-}=a_{01}^{+}=1$ and

$$
\begin{aligned}
a_{11}^{-} & =\frac{137(3 \pi-8)}{200(3 \pi+8)}, & b_{13}^{+} & =\frac{137 \pi \tilde{b}_{13}^{+}}{48 \cdot 10^{6}(3 \pi-8)^{2}(3 \pi+8)^{3}}, \\
b_{11}^{-} & =-\frac{274 \pi}{25(3 \pi+8)}, & b_{04}^{-} & =-\frac{\tilde{b}_{04}^{-}}{2304 \cdot 10^{6}(3 \pi-8)^{2}(3 \pi+8)^{3}}, \\
a_{11}^{+} & =-\frac{137(9 \pi+8)}{200(3 \pi+8)}, & a_{04}^{+} & =\frac{137 \pi \tilde{a}_{04}^{+}}{96 \cdot 10^{6}(3 \pi-8)^{2}(3 \pi+8)^{3}}, \\
a_{02}^{-} & =-\frac{137 \pi}{25(3 \pi+8)}, & b_{04}^{+} & =-\frac{\tilde{b}_{04}^{+}}{2304 \cdot 10^{6}(3 \pi-8)^{2}(3 \pi+8)^{3}}, \\
a_{12}^{+} & =\frac{18769 \pi(9 \pi-8)}{5000(3 \pi+8)^{2}(3 \pi-8)}, & b_{14}^{+} & =-\frac{18769 \pi \tilde{b}_{14}^{+}}{384 \cdot 10^{8}(3 \pi-8)^{2}(3 \pi+8)^{4}},
\end{aligned}
$$




$$
\begin{aligned}
b_{02}^{+} & =\frac{137 \pi}{50(3 \pi+8)}, & a_{05}^{-} & =-\frac{\tilde{a}_{05}^{-}}{442368 \cdot 10^{8}(3 \pi-8)^{3}(3 \pi+8)^{4}}, \\
b_{12}^{+} & =-\frac{56307 \pi\left(9 \pi^{3}-4 \pi^{2}-24 \pi-64\right)}{10^{4}(3 \pi+8)^{2}(3 \pi-8)}, & a_{05}^{+} & =-\frac{\tilde{a}_{05}^{+}}{442368 \cdot 10^{8}(3 \pi-8)^{3}(3 \pi+8)^{4}}, \\
a_{03}^{+} & =-\frac{18769 \pi\left(27 \pi^{3}-18 \pi^{2}-8 \pi-320\right)}{2 \cdot 10^{4}(3 \pi+8)^{2}(3 \pi-8)}, & b_{15}^{+} & =\frac{2571353 \pi \tilde{b}_{15}^{+}}{1536 \cdot 10^{11}(3 \pi-8)^{3}(3 \pi+8)^{5}}, \\
a_{13}^{+} & =\frac{137 \pi \tilde{a}_{13}^{+}}{8 \cdot 10^{6}(3 \pi-8)^{2}(3 \pi+8)^{3}}, & & a_{16}^{-}=-\frac{137 \tilde{a}_{16}^{-}}{55296 \cdot 10^{13}(3 \pi-8)^{4}(3 \pi+8)^{6}}, \\
b_{03}^{+} & =\frac{18769 \pi\left(3 \pi^{2}-19 \pi+24\right)}{5000(3 \pi+8)^{2}(3 \pi-8)}, & &
\end{aligned}
$$

with

$$
\begin{aligned}
& \tilde{a}_{05}^{-}=4181103066129489 \pi^{11}-81708322605754392 \pi^{10}+718656846864896880 \pi^{9} \\
& -3513609133038501120 \pi^{8}+14146051889489165568 \pi^{7}-52270826764050935424 \pi^{6} \\
& +137440270455671973888 \pi^{5}-224865343103126777856 \pi^{4} \\
& +223463238163167379456 \pi^{3}-117210783524524130304 \pi^{2} \\
& +19019943696196960256 \pi-17768647117686964224 \text {, } \\
& \tilde{a}_{16}^{-}=10387544938396777485 \pi^{14}-221600958685797919680 \pi^{13} \\
& +3266022594636620255520 \pi^{12}-30507673260011278331340 \pi^{11} \\
& +189967531652404749842256 \pi^{10}-801061043428012618757628 \pi^{9} \\
& +2321494974549119646057024 \pi^{8}-4908024571773961918342656 \pi^{7} \\
& +8188364135670255140327424 \pi^{6}-10698758352775621551112192 \pi^{5} \\
& +9974370209421370484588544 \pi^{4}-6632578667031793508548608 \pi^{3} \\
& +3115667460316900267917312 \pi^{2}-341925545063855526248448 \pi \\
& +356351263498044935503872 \text {, } \\
& \tilde{b}_{04}^{-}=16870647033 \pi^{8}-258683254506 \pi^{7}+887271066180 \pi^{6}+2964064868154 \pi^{5} \\
& -20822685722640 \pi^{4}+35660258560320 \pi^{3}-22653867737600 \pi^{2} \\
& +10756238168064 \pi-3269824380928 \text {, } \\
& \tilde{a}_{04}^{+}=41047803 \pi^{6}-401356296 \pi^{5}+2678981472 \pi^{4}-8009389248 \pi^{3}+8925785728 \pi^{2} \\
& -5674143744 \pi+7841538048 \text {, } \\
& \tilde{a}_{05}^{+}=4181103066129489 \pi^{11}-75051840112413912 \pi^{10}+590852382992759664 \pi^{9} \\
& -1912928489522952192 \pi^{8}+3518876760251069952 \pi^{7}-11356639358023421568 \pi^{6} \\
& +42263173441251182592 \pi^{5}-99395993027561435136 \pi^{4}+141659664965141463040 \pi^{3} \\
& -82254079608837636096 \pi^{2}-8065952330895327232 \pi-17768647117686964224 \text {, } \\
& \tilde{a}_{13}^{+}=13682601 \pi^{5}-34966647 \pi^{4}-86001900 \pi^{3}+76671376 \pi^{2}+406561536 \pi-126661632, \\
& \tilde{b}_{04}^{+}=16870647033 \pi^{8}-258683254506 \pi^{7}+1667069862372 \pi^{6}-790869101670 \pi^{5} \\
& -14311310178960 \pi^{4}+19927281672000 \pi^{3}+9234529541632 \pi^{2} \\
& -1824551743488 \pi-3269824380928 \text {, } \\
& \tilde{b}_{13}^{+}=41047803 \pi^{6}-474330168 \pi^{5}+2046541248 \pi^{4}-3441765408 \pi^{3}+2626609024 \pi^{2} \\
& -4636293120 \pi+5073936384 \text {, } \\
& \tilde{b}_{14}^{+}=369430227 \pi^{8}-6567648480 \pi^{7}+44634734748 \pi^{6}-133196235948 \pi^{5}+236392607904 \pi^{4} \\
& -375635258752 \pi^{3}+412507991040 \pi^{2}-220447137792 \pi+171924455424, \\
& \tilde{b}_{15}^{+}=49873080645 \pi^{11}-975295799280 \pi^{10}+11610242789130 \pi^{9}-73488565814220 \pi^{8} \\
& +274726689588612 \pi^{7}-667239956931456 \pi^{6}+1138618041586176 \pi^{5} \\
& -1556130962061312 \pi^{4}+1771316277657600 \pi^{3}-1168818168135680 \pi^{2} \\
& +228294320455680 \pi-167679759482880 \text {. }
\end{aligned}
$$

Then, system (12) has five hyperbolic limit cycles $\Gamma_{i}^{\varepsilon}, i=1, \ldots, 5$, for $\varepsilon$ small enough. Moreover, when $\varepsilon$ goes to 0 , the limit cycle $\Gamma_{i}^{\varepsilon}$ goes to the circle $x^{2}+y^{2}=i^{2}$, for $i=1, \ldots, 5$.

Proof. Using Proposition 2.5 we can obtain the functions $F_{i}^{ \pm}(r), h_{i}^{ \pm}(r, \theta), S_{i}^{ \pm}(r, \theta)$, for $i=1, \ldots, 6$ and, from Proposition 2.6 and Corollary 2.7, we can obtain the first coefficients of the series of $r_{i}^{ \pm}$for system (12). We have omitted the explicit expressions of these functions, due to the size of them. Thus, we get $M_{1}(\rho) \equiv M_{2}(\rho) \equiv M_{3}(\rho) \equiv M_{4}(\rho) \equiv M_{5}(\rho) \equiv 0$ and

$$
M_{6}(\rho)=\frac{137 \pi(\rho-1)(\rho-2)(\rho-3)(\rho-4)(\rho-5)}{120000(3 \pi+8) \rho^{4}} .
$$

Therefore, $\rho=1, \rho=2, \rho=3, \rho=4$, and $\rho=5$ are the five simple zeros of $M_{6}(\rho)$. The proof finishes in a similar way than Proposition 3.2 because all the zeros of $M_{6}$ are simple.

Proposition 4.1 provides the convergence of the difference map, rescaled by $\varepsilon^{6}$, to the function $M_{6}$, given in (13), when $\varepsilon$ goes to 0 . This fact is shown in Fig. 4, where the numerical approximation of the difference map is drawn together with the function $M_{6}$ for different 

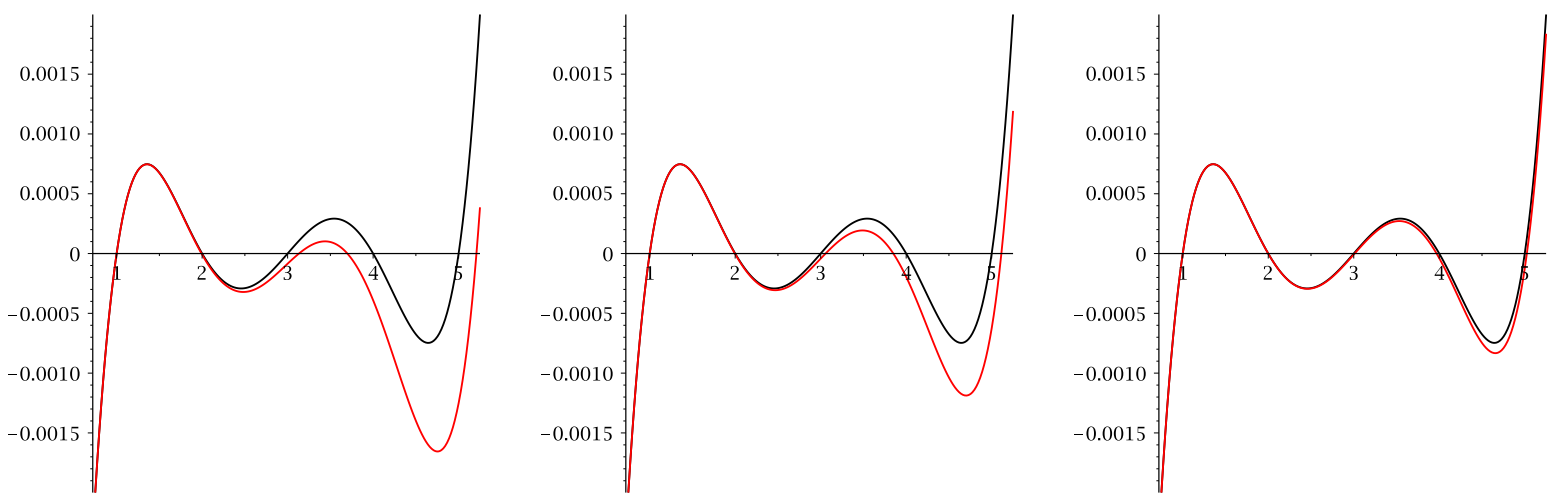

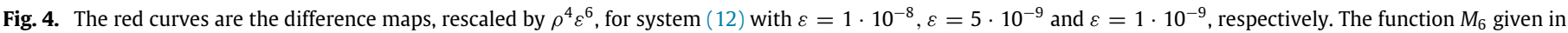
(13) is drawn in black. (For interpretation of the references to color in this figure legend, the reader is referred to the web version of this article.)
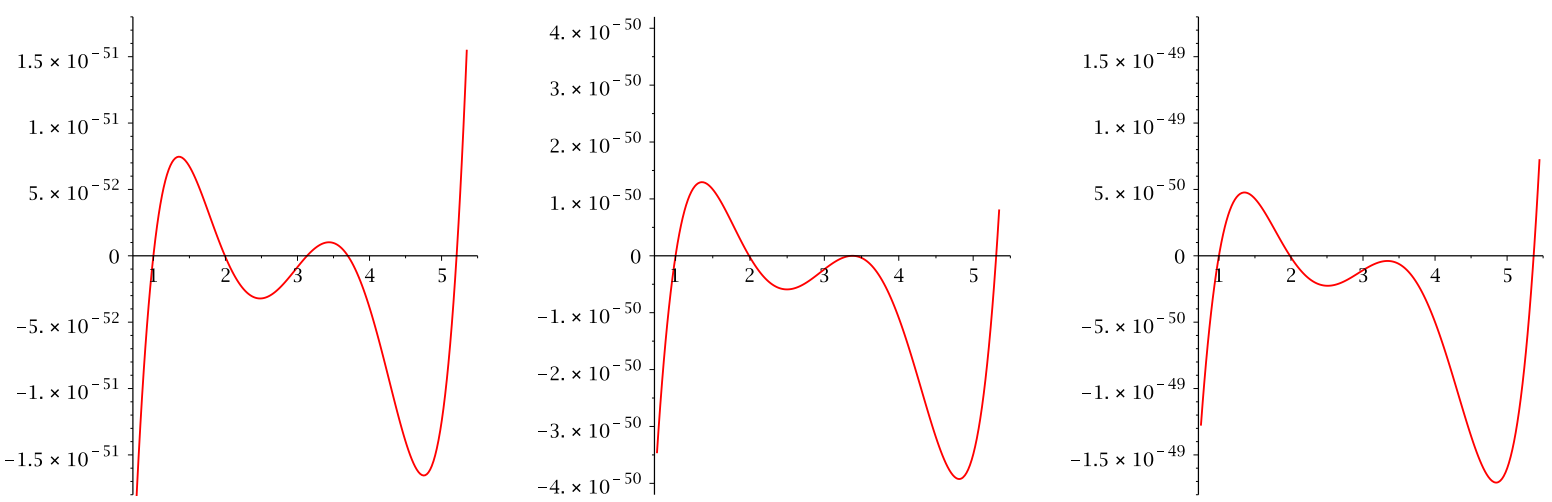

Fig. 5. Difference map for system (12), rescaled by $\rho^{4}$, with $\varepsilon=1 \cdot 10^{-8}, \varepsilon=\varepsilon_{s}^{+}$, and $\varepsilon=2 \cdot 10^{-8}$, respectively.
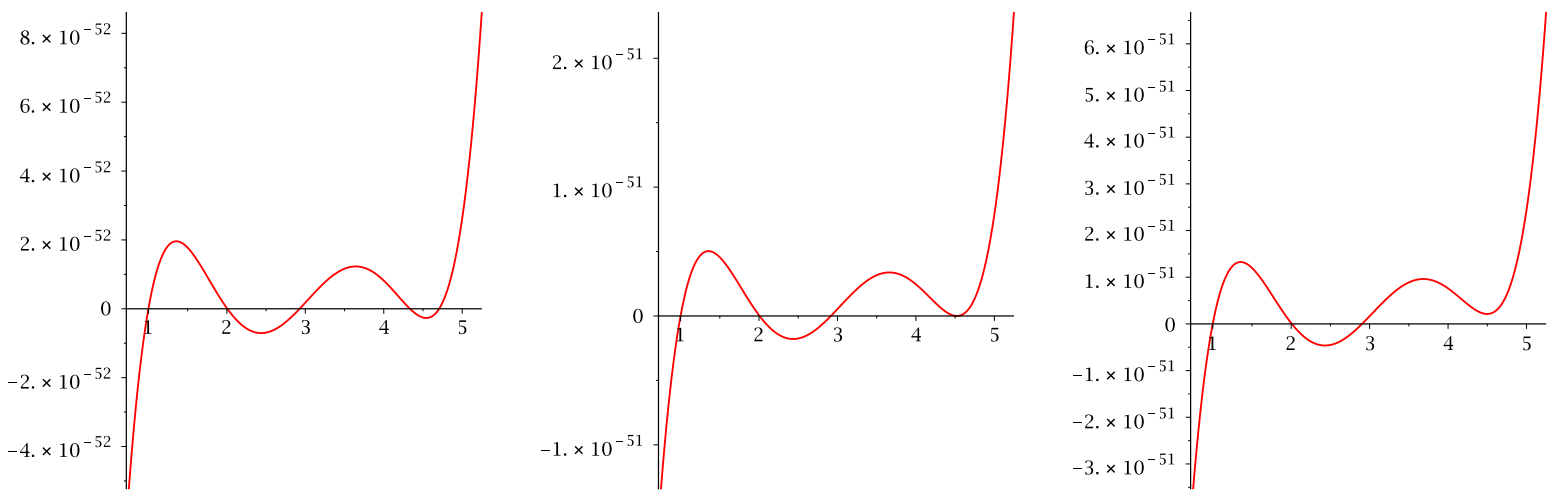

Fig. 6. Difference map for system (12), rescaled by $\rho^{4}$, with $\varepsilon=-8 \cdot 10^{-9}, \varepsilon=\varepsilon_{s}^{-}$, and $\varepsilon=-1.1 \cdot 10^{-8}$, respectively.

values of $\varepsilon$. We remark that the numerical computation must be done with high accuracy, since there are perturbation parameters of order $10^{-50}$.

Of course the coefficients given in above result are not very "friendly". Unfortunately, the vanishing conditions of first coefficients in $\varepsilon$ of the difference map imply intricate relations between the parameters of system.

The five limit cycles of Proposition 4.1 bifurcate from the level curves of the center for $\varepsilon$ small enough. These limit cycles exist until two of them collapse into a semistable one. This bifurcation can be seen in Figs. 5 and 6. Next proposition establishes the interval in $\varepsilon$ where the five limit cycles of system (12) exist. The proof is done looking for the first values of $\varepsilon$ where the difference map has a double zero.

Proposition 4.2. System (12) has five limit cycles when $\varepsilon \in\left(\varepsilon_{s}^{-}, 0\right) \cup\left(0, \varepsilon_{s}^{+}\right)$with $\varepsilon_{s}^{-} \approx-9.3602420168 \cdot 10^{-9}$ and $\varepsilon_{s}^{+} \approx 1.6090831394$. $10^{-8}$.

Finally we remark that, in this section, the computation of the difference map is obtained subtracting the evaluations of the analytical solution of system (12), in polar coordinates, in the values $\theta=\pi / 2$ and $\theta=-3 \pi / 2$. Consequently the numerical error only comes from the evaluation of this solution. 


\section{Piecewise Liénard linear systems}

In previous sections we have shown how the number of limit cycles bifurcating from the center increases with the perturbation order. When this growth is saturated, we can say that there has been a stabilization process. This section is devoted to study this phenomenon for some $\Sigma_{\alpha}$-piecewise linear Liénard systems. This stabilization process is similar to the one commented in [4], for $\Sigma_{\pi}$-piecewise linear systems. This phenomenon cannot be shown for the systems in Sections 3 and 4 due to the computational difficulties to get further in the order of perturbation.

\section{Proposition 5.1. For system}

$$
X^{ \pm}:(\dot{x}, \dot{y})=\left(-y+\sum_{i=1}^{N} \varepsilon^{i}\left(a_{0 i}^{ \pm}+a_{1 i}^{ \pm} x\right), x\right),
$$

the maximum number, $Z_{N}$, of zeros of the corresponding function $M_{N}(\rho)$ is $1,2,2,2,2,2$ when $N=1,2,3,4,5,6$. Moreover, for each $N$, there exist perturbation parameters such that system (14) exhibits $Z_{N}$ limit cycles for $\varepsilon$ small enough.

Proof. Considering the functions $P_{i}^{ \pm}(x, y)=a_{0 i}^{ \pm}+a_{1 i}^{ \pm} x$ and $Q_{i}^{ \pm}(x, y)=0$ as in the proof of Proposition 3.1, we get

$$
M_{1}(\rho)=\widehat{M}_{1}(\rho)=\left(a_{01}^{+}-a_{01}^{-}\right) \sin \alpha+\frac{1}{2}\left[\left(a_{11}^{+}-a_{11}^{-}\right)(\alpha+\sin \alpha \cos \alpha)+2 \pi a_{11}^{-}\right] \rho .
$$

In general, for $2 \leq i \leq 6$, the Poincaré-Pontryagin-Melnikov functions write as

$$
\widehat{M}_{i}(\rho)=\sum_{j=-1}^{1} C_{j, i}\left(\lambda_{i}\right) \rho^{j}, \quad \text { for } i=2,3, \quad \text { and } \quad \widehat{M}_{i}(\rho)=\sum_{j=-3}^{1} C_{j, i}\left(\lambda_{i}\right) \rho^{j}, \quad \text { for } i=4,6,
$$

where $C_{j, i}\left(\lambda_{i}\right)$ are polynomials of degree $i$ in the variables $\lambda_{i}=\left(a_{01}^{ \pm}, a_{11}^{ \pm}, \ldots, a_{0 i}^{ \pm}, a_{1 i}^{ \pm}\right)$. We have that $M_{1}(\rho) \equiv 0$ if and only if

$$
a_{01}^{-}=a_{01}^{+} \quad \text { and } \quad a_{11}^{+}=\frac{a_{11}^{-}(\alpha-2 \pi+\sin \alpha \cos \alpha)}{\alpha+\sin \alpha \cos \alpha} .
$$

Thus, the polynomials $C_{-1,2}, C_{0,2}$ and $C_{1,2}$ of the function $\widehat{M}_{2}(\rho)$ take the form

$$
\begin{aligned}
C_{-1,2}= & 0, \\
C_{0,2}= & \frac{\left(a_{02}^{+}-a_{02}^{-}\right)(\alpha+\sin \alpha \cos \alpha) \sin \alpha-2 \pi a_{11}^{-} a_{01}^{+}\left(1-\cos ^{3} \alpha\right)}{\alpha+\sin \alpha \cos \alpha}, \\
C_{1,2}= & \frac{-1}{2\left(\alpha^{2}+2 \alpha \sin \alpha \cos \alpha+\sin ^{2} \alpha \cos ^{2} \alpha\right)}\left[-4 \pi \alpha a_{12}^{-} \sin \alpha \cos \alpha\right. \\
& +2 \pi\left(a_{11}^{-}\right)^{2}\left(1-\cos ^{4} \alpha\right)(\alpha-\pi+\sin \alpha \cos \alpha)-2 \pi a_{12}^{-}\left(\alpha^{2}+\sin ^{2} \alpha \cos ^{2} \alpha\right) \\
& \left.+\left(a_{12}^{-}-a_{12}^{+}\right)\left(\alpha^{3}+\sin \alpha \cos \alpha\left(3 \alpha^{2}+3 \alpha \sin \alpha \cos \alpha+\sin ^{2} \alpha \cos ^{2} \alpha\right)\right)\right] .
\end{aligned}
$$

Solving $C_{0,2}=0$ and $C_{1,2}=0$ with respect to $a_{02}^{-}$and $a_{12}^{+}$, respectively, we get

$$
\begin{aligned}
a_{02}^{-}= & a_{02}^{+}-\frac{2 \pi a_{11}^{-} a_{01}^{+}\left(1-\cos ^{3} \alpha\right)}{(\alpha+\sin \alpha \cos \alpha) \sin \alpha} \\
a_{12}^{+}= & \frac{1}{\alpha^{3}+\sin \alpha \cos \alpha\left(3 \alpha^{2}+3 \alpha \sin \alpha \cos \alpha+\sin ^{2} \alpha \cos ^{2} \alpha\right)}\left[\alpha^{2} a_{12}^{-}(\alpha-2 \pi)\right. \\
& +a_{12}^{-}(3 \alpha-2 \pi) \sin ^{2} \alpha \cos ^{2} \alpha+a_{12}^{-} \sin \alpha \cos \alpha\left(\alpha(3 \alpha-4 \pi)+\sin ^{2} \alpha \cos ^{2} \alpha\right) \\
& \left.+2 \pi\left(a_{11}^{-}\right)^{2}\left(1-\cos ^{4} \alpha\right)(\alpha-\pi+\sin \alpha \cos \alpha)\right] .
\end{aligned}
$$

Then, the polynomial $C_{-1,3}$ of the function $\widehat{M}_{3}(\rho)$ becomes

$$
C_{-1,3}=\frac{2 \pi a_{11}^{-}\left(a_{01}^{+}\right)^{2}}{\alpha+\sin \alpha \cos \alpha}
$$

Note that $C_{-1,3} \equiv 0$ if and only if $a_{01}^{+}=0$ or $a_{11}^{-}=0$. We assume that the parameters $a_{01}^{-}, a_{11}^{+}, a_{02}^{-}$and $a_{12}^{+}$are like above. If $a_{01}^{+}=0$ we get $C_{-3,4}=C_{-2,4}=C_{-3,5}=C_{-2,5}=C_{-3,6}=C_{-2,6}=0$. This shows that the degree of the functions $\widehat{M}_{4}(\rho), \widehat{M}_{5}(\rho)$, and $\widehat{M}_{6}(\rho)$ is at most 2 . Therefore, when $N=2, \ldots, 6$, the maximum number of zeros of $\widehat{M}_{N}(\rho)$ is 2 . If $a_{11}^{-}=0$ we get $C_{-3,4}=C_{-2,4}=C_{-3,5}=C_{-3,6}=0$. In this case we should continue investigating the functions $\widehat{M}_{5}(\rho)$ and $\widehat{M}_{6}(\rho)$.

The polynomials $C_{0,3}$ and $C_{1,3}$ of the function $\widehat{M}_{3}(\rho)$ are given by

$$
\begin{aligned}
C_{0,3} & =\frac{\left(a_{03}^{-}-a_{03}^{+}\right)(\alpha+\sin \alpha \cos \alpha) \sin \alpha+2 \pi a_{01}^{+} a_{12}^{-}\left(1-\cos ^{3} \alpha\right)}{\alpha+\sin \alpha \cos \alpha}, \\
C_{1,3} & =\frac{1}{2}\left(a_{13}^{+}-a_{13}^{-}\right)(\alpha+\sin \alpha \cos \alpha)+\pi a_{13}^{-} .
\end{aligned}
$$


Solving $C_{0,3}=0$ and $C_{1,3}=0$ with respect to $a_{03}^{-}$and $a_{13}^{+}$, respectively, we get

$$
a_{03}^{-}=a_{03}^{+}-\frac{2 \pi a_{01}^{+} a_{12}^{-}\left(1-\cos ^{3} \alpha\right)}{(\alpha+\sin \alpha \cos \alpha) \sin \alpha} \quad \text { and } \quad a_{13}^{+}=\frac{a_{13}^{-}(\alpha-2 \pi+\sin \alpha \cos \alpha)}{\alpha+\sin \alpha \cos \alpha} \text {. }
$$

Then, the polynomial $C_{-1,4}$ of the function $\widehat{M}_{4}(\rho)$ becomes

$$
C_{-1,4}=\frac{2 \pi a_{12}^{-}\left(a_{01}^{+}\right)^{2} \sin \alpha}{\alpha+\sin \alpha \cos \alpha} .
$$

We have that $C_{-1,4} \equiv 0$ if and only if $a_{01}^{+}=0$ or $a_{12}^{-}=0$. In both cases, we get $C_{-2,5}=0$. This shows that the degree of $\widehat{M}_{5}(\rho)$ is at most two. Similar computations can be done for $\widehat{M}_{6}(\rho)$.

Consequently, when $N=1, \ldots, 6$, the maximum number of zeros of $\widehat{M}_{N}(\rho)$ is $1,2,2,2,2,2$, respectively. We remark that the zeros of $M_{N}(\rho)$ and $\widehat{M}_{N}(\rho)$ agree. Following the same ideas as in the proof of Proposition 3.2 explicit examples with 1 and 2 simple zeros can be found for orders $N=2, \ldots, 6$.

The proof concludes also using Proposition 2.1 to get the limit cycles corresponding to each simple zero of the function $M_{N}$.

Finally, we consider one more Liénard class. Now, the nonlinearity terms are in the second component while in the previous proposition they were in the first component. Clearly, the number of limit cycles is not the same and it points out that there is no symmetry between both problems. We omit the proof of the next result, because it follows using similar arguments and computations.

Proposition 5.2. Consider the system

$$
X^{ \pm}:(\dot{x}, \dot{y})=\left(-y, x+\sum_{i=1}^{N} \varepsilon^{i}\left(b_{0 i}^{ \pm}+b_{1 i}^{ \pm} x\right)\right)
$$

defined in $\Sigma_{\alpha}^{ \pm}$. Then, the maximum number, $Z_{N}$, of zeros of the corresponding function $M_{N}(\rho)$ is 1 when $N=1,2,3,4,5,6$. Moreover, for each $N$, there exist perturbation parameters such that system (15) exhibits $Z_{N}$ limit cycles for $\varepsilon$ small enough.

\section{Conclusions}

Planar piecewise linear differential systems with two zones were studied in this paper. We have seen that the separation line has a strong influence on the number of limit cycles that can appear. The simplest case occurs when the separation line is a straight line. For this case, it has already proven the existence of three limit cycles. It is worth to mention that it is still an open problem to determine whether three is the maximum number of limit cycles for $\Sigma_{\pi}$-planar piecewise linear differential systems.

If the separation line is no longer a straight line, more than three limit cycles can appear. In this paper we have considered the case when the two linear zones are angular sectors of angles $\alpha$ and $2 \pi-\alpha$, where $\alpha \in(0, \pi)$. That is the separation line $\Sigma_{\alpha}$ is formed by two semi straight lines with a breaking point at the origin. We have studied the bifurcation of limit cycles by studying higher order piecewise linear perturbations of a linear center. We have proved that the maximum number of limit cycles that can appear up to a sixth order is five. Moreover, this upper bound is reached for some values of $\alpha$.

Some natural questions still remain. The first one is to prove that there exist concrete examples with five limit cycles for every $\alpha \in(0, \pi)$. The second one is if the saturation is five and if it occurs for the sixth order. Finally, whether five is the maximum number of crossing limit cycles for the class of $\Sigma_{\alpha}$-planar piecewise linear differential systems.

\section{Acknowledgments}

The first author is supported by FAPESP Grant Number 2013/24541-0 and CAPES Grant Number 88881.030454/2013-01 Program CSF-PVE. The second author is supported by the MINECO/FEDER grants MTM2013-40998-P and UNAB13-4E-1604; and the AGAUR grant 2014 SGR568. All authors are partially supported by FP7-PEOPLE-2012-IRSES 316338 and 318999.

\section{References}

[1] A.A. Andronov, A.A. Vitt, S.E. Khaikin, Theory of Oscillators, Pergamon Press, Oxford-New York-Toronto, Ont, 1966, Translated from the Russian by F. Immirzi; Translation edited and abridged by W. Fishwick.

[2] A.F. Filippov, Differential Equations with Discontinuous Righthand Sides, in: Mathematics and its Applications (Soviet Series), vol. 18, Kluwer Academic Publishers Group, Dordrecht, 1988, Translated from the Russian.

[3] M. di Bernardo, C.J. Budd, A.R. Champneys, P. Kowalczyk, Piecewise-smooth Dynamical Systems, in: Applied Mathematical Sciences, vol. 163, Springer-Verlag London, Ltd., London, 2008, Theory and applications.

[4] C. Buzzi, C. Pessoa, J. Torregrosa, Piecewise linear perturbations of a linear center, Discrete Contin. Dyn. Syst. 33 (9) (2013) 3915-3936.

[5] S. Coombes, Neuronal networks with gap junctions: a study of piecewise linear planar neuron models, SIAM J. Appl. Dyn. Syst. 7 (3) (2008) 1101-1129.

[6] F. Giannakopoulos, K. Pliete, Planar systems of piecewise linear differential equations with a line of discontinuity, Nonlinearity 14 (6) (2001) 1611-1632.

[7] M. Han, W. Zhang, On Hopf bifurcation in non-smooth planar systems, J. Differential Equations 248 (9) (2010) 2399-2416.

[8] S.-M. Huan, X.-S. Yang, On the number of limit cycles in general planar piecewise linear systems, Discrete Contin. Dyn. Syst. 32 (6) (2012) $2147-2164$.

[9] R.I. Leine, D.H. van Campen, Discontinuous bifurcations of periodic solutions, Math. Comput. Modelling 36 (3) (2002) 259-273. Mathematical modelling of nonlinear systems (Leeds, 1999).

[10] J. Llibre, E. Ponce, Three nested limit cycles in discontinuous piecewise linear differential systems with two zones, Dyn. Contin. Discrete Impuls. Syst. Ser. B Appl. Algorithms 19 (3) (2012) 325-335.

[11] J. Llibre, M.A. Teixeira, J. Torregrosa, Lower bounds for the maximum number of limit cycles of discontinuous piecewise linear differential systems with a straight line of separation, Internat. J. Bifur. Chaos Appl. Sci. Engrg. 23 (4) (2013) 1350066

[12] Y. Tian, P. Yu, Center conditions in a switching Bautin system, J. Differential Equations 259 (3) (2015) 1203-1226. 


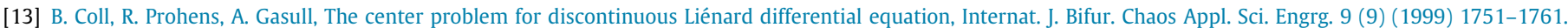
Discrete dynamical systems.

[14] E. Freire, E. Ponce, F. Torres, The discontinuous matching of two planar linear foci can have three nested crossing limit cycles, Publ. Mat. 58 (suppl.) (2014) $221-253$.

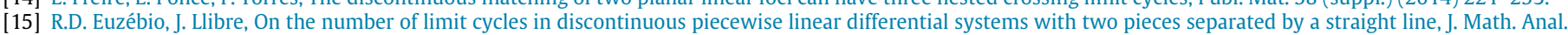
Appl. 424 (1) (2015) 475-486.

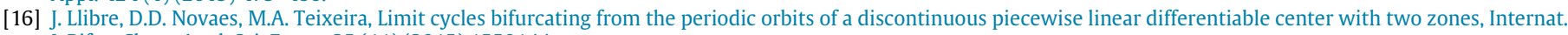
J. Bifur. Chaos Appl. Sci. Engrg. 25 (11)(2015) 1550144.

[17] J. Llibre, D.D. Novaes, M.A. Teixeira, Maximum number of limit cycles for certain piecewise linear dynamical systems, Nonlinear Dynam. 82 (3) (2015) $1159-1175$.

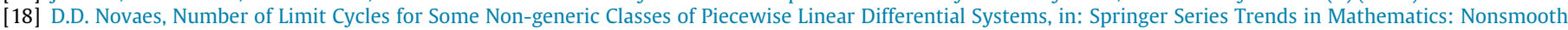
Dynamics, 2016, in press.

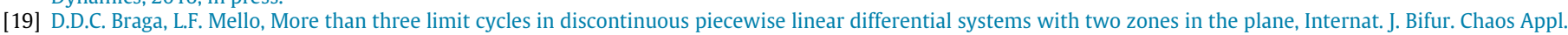
Sci. Engrg. 24 (4) (2014) 1450056.

[20] D.D. Novaes, E. Ponce, A simple solution to the Braga-Mello conjecture, Internat. J. Bifur. Chaos Appl. Sci. Engrg. 25 (1) (2015) 1550009.

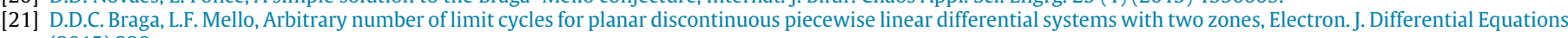
(2015) 228.

[22] H. Giacomini, J. Llibre, M. Viano, On the nonexistence, existence and uniqueness of limit cycles, Nonlinearity 9 (2) (1996) 501-516.

[23] I.D. Iliev, The number of limit cycles due to polynomial perturbations of the harmonic oscillator, Math. Proc. Cambridge Philos. Soc. 127 (2) (1999) 317-322.

[24] A. Gasull, J. Torregrosa, A relation between small amplitude and big limit cycles, Rocky Mountain J. Math. 31 (4) (2001) 1277-1303.

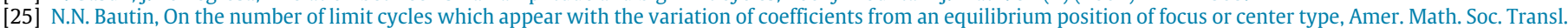
1954 (100) (1954) 19.

[26] J.P. Françoise, Successive derivatives of a first return map, application to the study of quadratic vector fields, Ergodic Theory Dynam. Systems 16 (1) (1996) 87-96.

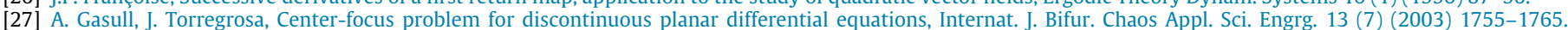
Dynamical systems and functional equations (Murcia, 2000).

[28] I.D. Iliev, L.M. Perko, Higher order bifurcations of limit cycles, J. Differential Equations 154 (2) (1999) $339-363$.

[29] R. Prohens, J. Torregrosa, Shape and period of limit cycles bifurcating from a class of Hamiltonian period annulus, Nonlinear Anal. 81 (2013) $130-148$.

[30] R. Prohens, J. Torregrosa, Periodic orbits from second order perturbation via rational trigonometric integrals, Physica D 280/281 (2014) 59-72.

[31] Maple 18. Maplesoft, a division of Waterloo Maple Inc., Waterloo, Ontario.

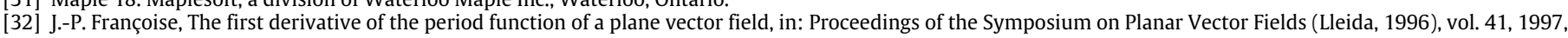
pp. $127-134$ 